\title{
ASSOCIATED PRODUCTION OF AN ISOLATED, LARGE-TRANSVERSE-MOMENTUM LEPTON (ELECTRON OR MUON), AND TWO JETS AT THE CERN p $\bar{p}$ COLLIDER
}

\author{
UA1 Collaboration, CERN, Geneva, Switzerland
}

Aachen ${ }^{1}-$ Annecy $(\text { LAPP })^{2}-$ Birmingham $^{3}-\mathrm{CERN}^{4}-$ Harvard $^{5}-\mathrm{Helsink}^{6}-\mathrm{Kiel}^{7}-$ Queen Mary College, London ${ }^{8}-$ NIKHEF, Amsterdam ${ }^{9}-$ Paris (Coll. de France $)^{10}-$ Riverside ${ }^{11}-$ Rome $^{12}-$ Rutherford Appleton Lab. ${ }^{13}$-Saclay $(\mathrm{CEN})^{14}-$ Vienna ${ }^{15}$ - Wisconsin ${ }^{16}$ Collaboration

G. Arnison ${ }^{13}$, O.C. Alikofer ${ }^{7}$, A. Astbury ${ }^{13 *}$, B. Aubert ${ }^{2}$, C. Bacci ${ }^{12}$, G. Bauer ${ }^{16}$, A. Bézaguet ${ }^{4}$, R.K. Bock ${ }^{4}$, T.J.V. Bowcock ${ }^{8}$. M. Calvetti ${ }^{4}$, P. Cat $\chi^{2}$, P.Cennini ${ }^{4}$, S. Centro ${ }^{+}$, F. Ceradini ${ }^{12}$, S. Cittolin ${ }^{4}$, D. Cline ${ }^{16}$. C. Cochet ${ }^{14}$, J. Colas ${ }^{2}$, M. Corden ${ }^{3}$, D. Dallman ${ }^{4,15}$, D. Dau ${ }^{4.7}$, M. DeBeer ${ }^{14}$, M. Della Negra ${ }^{2,4}$, M. Demoulin ${ }^{4}$, D. Denegri ${ }^{14}$, D. DiBitonto ${ }^{4}$, A. Di Ciaccio ${ }^{12}$, L. Dobrzynski ${ }^{10}$, J.D. Dowell ${ }^{3}$, K. Eggert ${ }^{1}$, E. Eisenhandler ${ }^{8}$, N. Ellis ${ }^{4}$, P. Erhard ${ }^{1}$, H. Faissner $^{1}$.

M. Fincke ${ }^{3 *}$, P. Flynn ${ }^{13}$. G. Fontaine ${ }^{10}$, R. Frey ${ }^{11}$, R. Frühwirth ${ }^{15}$, J. Garvey ${ }^{3}$, S. Geer ${ }^{5}$.

C. Ghesquière ${ }^{10}$, P. Ghez ${ }^{2}$, W.R. Gibson ${ }^{8}$, Y. Giraud-Héraud ${ }^{10}$, A. Givernaud ${ }^{14}$, A. Gonidec ${ }^{2}$.

G. Grayer ${ }^{13}$, T. Hansl-Kozaneck a ${ }^{1}$. W.J. Haynes ${ }^{13}$, L.O. Hertzberger ${ }^{9}$, D. Hoffmann ${ }^{1}$.

H. Hoffmann" ${ }^{4}$ D.J. Holthuizen ${ }^{9}$, R.J. Homer ${ }^{3}$, A. Honma ${ }^{8}$, W. Jank ${ }^{4}$, G. Jorat ${ }^{4}$, P.I.P. Kalmus ${ }^{8}$, V. Karimäki ${ }^{6}$, R. Keeler ${ }^{8 *}$, I. Kenyon ${ }^{3}$, A. Kernan ${ }^{11}$, R. Kinnunen ${ }^{6}$, W. Kozanecki ${ }^{11}$, D. Kryn ${ }^{4} \cdot 10$,

P. Kyberd ${ }^{8}$, F. Lacava ${ }^{12}$, J.-P. Laugier ${ }^{14}$, J.-P. Lees ${ }^{2}$, H. Lehmann ${ }^{1}$, R. Leuchs ${ }^{7}$, A. Lévêque ${ }^{4}$.

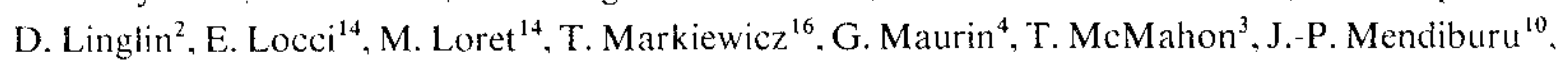
M.-N. Minard ${ }^{2}$, M. Mohammadi ${ }^{16}$. M. Moricca ${ }^{12}$, K. Morgan $^{11}$, F. Muller ${ }^{4}$, A.K. Nandi ${ }^{8}$, L. Naumann ${ }^{4}$, A. Norton ${ }^{4}$. A. Orkin-Lecourtois ${ }^{10}$, L. Paoluzi ${ }^{12}$, F. Pauss ${ }^{4}$, G. Piano Mortari ${ }^{12}$, E. Pietarinen ${ }^{6}$, M. Pimiä ${ }^{6}$, D. Pitman ${ }^{11}$, A. Placci ${ }^{4}$, J.-P. Porte ${ }^{4}$, E. Radermacher ${ }^{1}$, J. Ransdell ${ }^{11}$, H. Reithler ${ }^{1}$, J.-P. Revol ${ }^{4}$, J. Rich ${ }^{14}$, M. Rijssenbeek ${ }^{4}$, C. Roberts $^{13}$, J. Rohlf ${ }^{5}$, P. Rossi ${ }^{4}$, C. Rubbia ${ }^{4.5}$, B. Sadoulet ${ }^{4}$, G. Sajot ${ }^{10}$, G. Salvini ${ }^{12}$, J. Sass ${ }^{14}$, A. Savoy-Navarro ${ }^{14}$, D. Schinzel ${ }^{4}$. W. Scott ${ }^{13}$, T.P. Shah ${ }^{13}$, I. Sheer ${ }^{11}$, D. Smith ${ }^{11}$, M. Spiro ${ }^{14}$, J. Strauss ${ }^{15}$, J. Streets ${ }^{3}$, K. Sumorok ${ }^{4}$. F. Szoncso ${ }^{15}$, C. Tao ${ }^{10}$, G. Thompson ${ }^{8}$, J. Timmer ${ }^{4}$, E. Tscheslog ${ }^{1}$, J. Tuominiemi ${ }^{6}$, B. van Eijk ${ }^{9}$, J.-P. Vialle ${ }^{2}$, J. Vrana ${ }^{10}$, V. Vuillemin ${ }^{4}$, H.D. Wahl ${ }^{15}$, P. Watkins ${ }^{3}$, J. Wilson $^{3}$, I. Wingerter ${ }^{2}$. C.-E. Wuiz ${ }^{15}$ and M. Yvert ${ }^{2}$

(Submitted to Physics Letters B)

* Now at University of Victoria, Canada.

+ Visitor from the University of Padua. Italy. 


\begin{abstract}
A clear signal is observed for the production of an isolated large-transverse-momentum lepton in association with two or three centrally produced jets. The two-jet events cluster around the $\mathrm{W}^{ \pm}$mass, indicating a novel decay of the Intermediate Vector Boson. The rate and features of these events are not consistent with expectations of known quark decays (charm, bottom). They are, however, in agreement with the process $\mathrm{W} \rightarrow t \bar{b}$ followed by $t \rightarrow b \ell v$, where $t$ is the sixth quark (top) of the weak Cabibbo current. If this is indeed so, the bounds on the mass of the top quark are $30 \mathrm{GeV} / \mathrm{c}^{2}<\mathrm{m}_{\mathrm{t}}<50 \mathrm{GeV} / \mathrm{c}^{2}$.
\end{abstract}




\section{INTRODUCTION}

The UA1 Collaboration has recorded 68 events with $\mathrm{e}^{ \pm} y_{\mathrm{e}}$ decays [1] of the charged Intermediate Vector Boson (IVB), and a more restrictive sample of $\mathrm{W}^{ \pm} \rightarrow \mu^{ \pm} v_{\mu}$ decays [2] (14 events). These events have a remarkably clean signature: the main kinematical effect of the composite nature of the initial hadrons is limited to a longitudinal motion and a transverse momentum given to the IVB. Occasionally, the emission of one or more QCD jets radiated from the incident partons is also observed [3]. In agreement with expectations for initial state bremsstrahlung, these jets are emitted primarily along the incoming beam directions and have a rapidly falling transverse energy distribution.

Observation of the electronic and muonic decay modes of the IVB has provided an understanding of the production process and has built up confidence in the apparatus necessary to extend the search to the quark decay modes. The structure of the weak current responsible for the hadronic decays of the IVBs is expected to be coupled to charge $2 / 3$ quarks and to the Cabibbo-rotated states $d_{c}, s_{c}, b_{c}$ (namely, linear combinations of charge $1 / 3$ quarks) with the same strength as the coupling to leptons. From the number of observed leptonic decays, taking into account detection efficiencies and colour factor, we conclude that $(255 \pm 30) \mathrm{W}^{ \pm} \rightarrow \mathrm{ud}_{\mathrm{c}}$ and the same number of $\mathrm{W}^{ \pm} \rightarrow \mathrm{cs}_{\mathrm{c}}$ must also have been produced in the same data sample. Unfortunately, the observation of these decays is made very difficult by the presence of a relatively large QCD jet background.

The present paper deals with the search for the sixth quark, the t-quark (top or truth), which completes the family of the weak currents with a third doublet $\left(t \bar{b}_{c}\right)$ which manifests itself in the decay $\mathrm{W}^{+} \rightarrow t \overline{\mathrm{b}}_{\mathrm{c}}$ (and also $\mathrm{W}^{-} \rightarrow \overline{\mathrm{t}}_{\mathrm{c}}$ ), provided $\mathrm{m}_{\mathrm{t}}<\mathrm{m}_{\mathrm{W}}-\mathrm{m}_{\mathrm{b}}$. Previous, unsuccessful searches for the t-quark in $\mathrm{e}^{+} \mathrm{e}^{-}$colliding beams [4] have established a mass limit $\mathrm{m}_{\mathrm{t}} \geq 22 \mathrm{GeV} / \mathrm{c}^{2}$. Using the available energy in the decay $\mathrm{W}^{ \pm} \rightarrow \mathrm{tb}_{\mathrm{c}}$, we can extend the search up to masses of about $65 \mathrm{GeV} / \mathrm{c}^{2}$, since the $t$-quark is produced in association with a relatively light quark. For $m_{t}=40 \mathrm{GeV} / \mathrm{c}^{2}$ the reduction factor with respect to massless quarks is 0.71 , giving, before detection cuts, an expectation of $(181 \pm 20) \mathrm{W}^{ \pm} \rightarrow \mathrm{tb}_{\mathrm{c}}$ decays from our integrated luminosity.

We concentrate on the semileptonic decay channels of the t-quark:

$$
\mathrm{W}^{+} \rightarrow \mathrm{t}_{\mathrm{c}}\left(\mathrm{t} \rightarrow \ell^{+} v \mathrm{~b}_{\mathrm{c}}\right) \quad \ell \equiv \text { (electron, muon) }
$$

(and the corresponding reaction for $\mathrm{W}^{-}$). In spite of the smaller number of events due to the additional semileptonic branching ratio, this channel is chosen for the clean signature it provides, i.e. two jets, a lepton, and some missing transverse energy (v) [5]. These events have several features which permit them to be identified and separated from other sources of background:

i) The invariant mass of the $\left(b_{c} \bar{b}_{c} \ell v\right)$ system must peak around the $W$ mass. Replacing the neutrino momentum $\vec{p}_{v}$ by the measured missing transverse energy $\Delta \vec{E}_{m}[5]$ broadens the peak somewhat, with a small shift of the average mass value.

ii) The invariant mass of the $\left(b_{c} \ell v\right)$ system must be compatible with a common value, namely $m_{t}$ [6] (to a good approximation after the substitution $\vec{p}_{v} \rightarrow \Delta \overrightarrow{\mathrm{E}}_{\mathrm{m}}$ ).

iii) The t-quark is heavy $\left(\mathrm{m}_{\mathrm{t}} \geq 22 \mathrm{GeV} / \mathrm{c}^{2}\right)$ and therefore relatively slow in the laboratory. Angles between particles from the decay in the laboratory are very wide. Furthermore, the lepton momentum has a large component normal to the $b_{c}$ jet, $p_{n}$. In the case of decays of lighter quarks $(b, c), p_{n} \lesssim\left(m_{b} / 2, m_{c} / 2\right)$.

iv) The recoiling $\bar{b}_{c}$ jet has a characteristic Jacobian peak in the transverse energy distribution, which makes it possible, in most cases, to distinguish it from the other, lower-energy $b_{c}$ jet.

As discussed in detail further on, a simple set of topological cuts on the event configuration enables us to extract an essentially background-free event sample. However, the number of 
surviving events is also considerably reduced. For a semileptonic branching ratio $\sim 1 / 9$ ('naïve' prediction based on lepton and quark counting) and $m_{t}=40 \mathrm{GeV} / \mathrm{c}^{2}$, we expect $(20 \pm 2.2)$ electrons (both signs) and an equal number of muons. With reasonable cuts on the transverse energy of jets and leptons $\left[\mathrm{E}_{\mathrm{T}}\left(\overline{\mathrm{b}}_{\mathrm{c}}\right)>8 \mathrm{GeV} ; \mathrm{E}_{\mathrm{T}}\left(\mathrm{b}_{\mathrm{c}}\right)>7 \mathrm{GeV} ; \mathrm{p}_{\mathrm{T}}(\ell)>12 \mathrm{GeV} / \mathrm{c}\right]$ we arrive at $(4 \pm 0.3)$ events for each leptonic channel, before geometrical and track isolation cuts.

Searching for this small number of events deserves some remarks:

i) Both the muon and the electron samples will be used in order to increase the significance of the result. Evidence of an effect must rely on its independent observation in both decay channels.

ii) The electron and muon identification must be considerably improved with respect to the previous search for leptonic IVB decays, since now the signal is only $\sim 1 / 20$ of the $\mathrm{W} \rightarrow \ell+v$ rate and the average lepton energy is a factor of $\sim 3$ smaller, which greatly enhances the probability of hadrons simulating the leptonic signature. Furthermore, the event topology requires a dominant jet activity, thus enhancing QCD associated backgrounds.

Finally, a residual leptonic signal due to $\mathrm{W} \rightarrow t \bar{b}_{c}$ decays must be clearly separated from production of $(b \bar{b})$ and $(c \bar{c})$ with subsequent associated semileptonic decays, which are copious sources of leptons and jets. The cross-section and kinematics of heavy quark pair-production via gluon-related and quark-related strong interaction graphs are relatively poorly known at collider energies. In order to extract from the data the information needed to reliably evaluate the expected background from this source, a parallel analysis has been performed aimed at strong interaction production of heavy quarks.

\section{THE ELECTRON SAMPLE}

As described extensively in previous publications $[1,7]$ to which we refer the reader for details, after momentum (p) determination from the magnetic curvature measured by the central detector, electron identification is based on absorption in the $27 \mathrm{X}_{0}$ of a $4 \pi$ lead/scintillator calorimeter hodoscope segmented four times in depth $\left(3 \mathrm{X}_{0}, 7 \mathrm{X}_{0}, 10 \mathrm{X}_{0}, 7 \mathrm{X}_{0}\right)$, followed by a hadron calorimeter in which only a small residual energy $E_{h a d}$ is expected. Each of the four segments of the lead/scintillator calorimeter cells is read out by four independent photomultipliers in a way that permits the determination, by pulse division, of the centroid of the energy deposition in two orthogonal directions.

In the previously reported observation of the $\mathrm{W}^{ \pm} \rightarrow \mathrm{e}^{ \pm} \boldsymbol{v}_{\mathrm{e}}$ decay [1], very generous selection criteria were sufficient to obtain an essentially pure event sample, namely: i) a charged track of $\mathrm{p}_{\mathrm{T}}>7 \mathrm{GeV}$, of projected length $>30 \mathrm{~cm}$ and with at least 20 digitizings; ii) an energy deposition of $\mathrm{E}_{\mathrm{T}}>15 \mathrm{GeV}$ in two adjacent e.m. cells; iii) a match within 5 st. dev. between the impact of the track and the centroid of the energy depositions in the calorimeter; iv) an energy deposition $\mathrm{E}_{\text {had }}<$ $600 \mathrm{MeV}$ in the subsequent hadronic calorimeter; v) electron isolation, namely no more than $10 \%$ of the electron energy is allowed for any additional energy deposited in a cone around the electron track $\Delta \mathrm{R} \equiv\left(\Delta \eta^{2}+\Delta \phi^{2}\right)^{\frac{1}{2}} \leq 0.7$, where $\phi$ is the azimuthal angle measured in radians and $\eta$ is the rapidity; and vi) no jet back-to-back in $\phi$ with respect to the electron within $\pm 30^{\circ}$. In this way, we have detected $49 \mathrm{~W}^{ \pm} \rightarrow \mathrm{e}^{ \pm} v_{\mathrm{e}}$ decays, completely background free and satisfying the additional condition [5] $\Delta E_{m}>15 \mathrm{GeV}$. These events give us an ideal electron calibration sample for the present search.

However, as soon as the limitation on the jet activity (vi) and the missing-energy requirements are dropped, we find a much larger sample of presumably heavily contaminated events. Requiring the electron transverse energy $E_{T}>12 \mathrm{GeV}$, and tightening the $E_{\text {had }}$ condition to $E_{\text {had }}<200 \mathrm{MeV}$ leaves us with as many as 152 events. A first reduction of the sample can be achieved by removing photon conversions in the beam tube and in the walls of the central detector. These events can easily 
be recognized, by scanning or program, by looking for tracks which have a small minimum distance $\mathrm{D}$ from the electron track. As one can see from figs. $1 \mathrm{a}$ and $\mathrm{lb}$, there is a large peak centred around $\mathrm{D}=0$, mostly from track pairs having charges of opposite signs. Applying a cut on $\mathrm{D}$ at $3 \sigma$, forty-three conversions are removed. Recognition of conversions by program and by scanning agree very well, and the number of conversion events is in agreement with expectations [8], based on the flux of high-energy $\pi^{0}$ 's and the amount of material traversed.

The remaining 109 events are still largely contaminated by multiple particle overlaps, namely jets with one charged energetic hadron and one or several $\pi^{0 \%}$ s simulating the e.m. behaviour. In order to eliminate this background, we raise the electron transverse energy threshold to $15 \mathrm{GeV}$ and make use of the full rejection power of the detector, namely: i) a good match between the momentum measurement and the energy deposition in the calorimeters, $|(1 / \mathrm{p})-(1 / \mathrm{E})|<3 \sigma$; ii) a good electromagnetic shape in the energy depositions of the four e.m. segments, characterized [9] by $\chi_{\mathrm{R}}^{2}$ $<20$; and iii) a stricter isolation requirement for the electron track, namely that the $\Sigma \mathrm{p}_{\mathrm{T}}$ of all other tracks and the energy deposited in all calorimeters $\Sigma \mathrm{E}_{\mathrm{T}}$ be less than $1 \mathrm{GeV}$ in a cone of $\Delta \mathrm{R} \leq 0.4$ around the track. This new selection leads to twelve events, namely seven events with electrons and one jet and five events with at least two jets. Forty-four out of the forty-nine $\mathrm{W}$ events survive these cuts. One can compare the distributions of $\Sigma E_{T}$ versus $\chi_{R}^{2}$ for the calibration sample of $W^{ \pm} \rightarrow$ $\mathrm{e}^{ \pm} v_{\mathrm{e}}$ decays (fig. $2 \mathrm{a}$ ), the sample of single jets (fig. $2 b$ ), and the sample of events with at least two jets (fig. 2c). Whilst both the $\geq 2$ jet events and $\mathrm{W} \rightarrow \mathrm{e}^{\ddagger} v_{\mathrm{e}}$ events have a cleanly isolated electron and small values of $\chi_{\mathrm{R}}^{2}$, the single-jet events are more widely distributed, and indeed their precise number depends on the choice of our cuts, indicating that in general they are not truly isolated. We concentrate on the five events with $\geq 2$ jets. Their main parameters are listed in tables $1 \mathrm{a}$ and $1 \mathrm{c}$. Their electron properties closely resemble the ones of the W calibration sample (fig. 3).

Next we shall evaluate the expected background. As already pointed out, the dominant background is expected to come from QCD jets faking electrons by fragmenting, such that one energetic charged pion overlaps with one or more neutral pions. Two methods have been used to estimate this background: i) a global method, using a $\pi^{0}+\geq 2$ jet data sample, in which the shape of the expected QCD background distribution is compared with the shape of the corresponding distribution for the candidate events; ii) a direct method in which the absolute rate of $\left(\pi^{ \pm}+n \pi^{0}\right)+$ jet events is extracted from a $\pi^{ \pm}+$jet selection. The probability that the selected $\pi^{ \pm}$pass the isolated electron selection criteria is then folded into the resulting estimated background rate.

The shape of the QCD background from $\pi^{0}+\geq 2$ jet events is shown in fig. $4 \mathrm{a}$, in which $\mathrm{E}_{\mathrm{T}}^{\text {out }}$, the transverse energy component of the isolated $\pi^{0}$ perpendicular to the plane formed by the $\mathrm{p} \overrightarrow{\mathrm{p}}$ axis and the highest $E_{\mathrm{T}}$ jet $\left(\mathrm{j}_{1}\right)$ is plotted as a function of $\cos \theta_{\mathrm{j}_{2}}^{*}$. The angle $\theta_{\mathrm{j}_{2}}^{*}$ is between the average $(\mathrm{p} \overline{\mathrm{p}})$ beam axis and the lowest $E_{T}$ jet $\left(j_{2}\right)$ in the $\left(\pi^{0} j_{1} j_{2}\right)$ rest frame. The five electron $+\geq 2$ jet events (fig. 4b) are all contained within a region $\mathrm{RI}=\left(\mathrm{E}_{\mathrm{T}}^{\text {out }}>8 \mathrm{GeV},\left|\cos \theta_{\mathrm{j}_{2}}^{*}\right|<0.73\right.$ ), whilst the majority of background QCD events lie in the complementary region RII [10]. Table 2 summari:.es the number of events in these two regions. On the basis of these statistics the probability that the QCD background events have a distribution identical to the five electron $+\geq 2$ jet events is $8 \times$ $10^{-5}$, i.e. a $4 \sigma$ difference in shape. The choice of regions RI and RII, however, is arbitrary and a comparison of shape with the Kolomogorov test gives a probability of $5.2 \times 10^{-4}$, i.e. a $3.5 \sigma$ difference.

To determine the absolute magnitude of the background from $\pi^{ \pm}+\geq 2$ jet events, we estimate the probability that a $\pi^{ \pm}$satisfies the isolated electron selection criteria. Of the $169 \pi^{ \pm}+\geq 2$ jet events originally selected, 68 satisfy the electron trigger requirement of $\mathrm{E}_{\mathrm{T}}>12 \mathrm{GeV}$. The probability that these charged pions fake an isolated electron is estimated to be $1.5 \times 10^{-3}$, yielding a total of 0.1 background events [11]. Results of this background calculation are given in table $3 \mathrm{a}$. The corresponding number of background events in region $\mathrm{RI}$ is less than 0.06 . 
Finally the background from unseen conversions with one electron triggered with $\mathrm{p}_{\mathrm{T}}>$ $15 \mathrm{GeV} / \mathrm{c}$ and the other electron unobserved $\left(\mathrm{p}_{\mathrm{T}}<0.05 \mathrm{GeV} / \mathrm{c}\right)$ is less than 0.02 events, determined from the measured $\pi^{0}$ flux in region RI. Therefore, we conclude that the five events with at least two jets have genuine electrons.

\section{THE MUON SAMPLE}

A simple set of cuts is used to arrive at a sample of isolated muons with one or more jets: i) a muon track in the central detector with $\mathrm{p}_{\mathrm{T}}^{(\mu)}>12 \mathrm{GeV} / \mathrm{c}$, good geometrical match in the muon chambers, track projected length $\geq 40 \mathrm{~cm}$, and at least 30 digitizings; ii) isolation, namely $\Sigma \mathrm{p}_{\mathrm{T}}<$ $0.1 \mathrm{p}_{\mathrm{T}}^{(\mu)}, \Sigma \mathrm{E}_{\mathrm{T}}<0.2 \mathrm{p}_{\mathrm{T}}^{(\mu)}$, where the sum extends to all tracks and calorimeter hits in a cone $\Delta \mathrm{R} \leq$ 0.4 . Forty events survive these cuts. The dominant source of background comes from the decays of pions and kaons in the central detector drift volume. In the case of slow kaons, parent and daughter tracks may form a kink in the track digitizings in a configuration which is reconstructed as a single fake high-momentum track. After visual scanning and rejection of obvious $\mathrm{K} \rightarrow \mu v$ decays in flight within the central detector volume (kinks), and an enhanced isolation requirement of no jet within a distance $\Delta R=1$ from the muon, we are left with twelve events, seven with one jet, four with two jets, and one with three jets. Again we concentrate on the $\geq 2$ jet events, which are listed in table $1 \mathrm{~b}$ and $1 \mathrm{c}$. The residual decay background has been determined from a $5 \mathrm{nb}^{-1}$ data sample collected with a low threshold $\left(\mathrm{E}_{\mathrm{T}}>15 \mathrm{GeV}\right)$ jet trigger. This trigger requirement is satisfied by the five muon $+\geq 2$ jet events. We wish to estimate the probability that decaying hadrons $(\pi, \mathrm{K} \rightarrow \mu v)$ pass our track-quality cuts and are reconstructed with $p_{\mathrm{T}}>12 \mathrm{GeV} / \mathrm{c}$. The final background rate is then the convolution of this probability (per decaying hadron) with the measured $\mathrm{p}_{\mathrm{r}}$ spectrum of the $5 \mathrm{nb}^{-1}$ sample. For a mixture of $50 \%$ pions and $25 \%$ kaons, this probability is typically $4 \times 10^{-5}$ for a hadron with a $\mathrm{p}_{\mathrm{T}}$ of $8 \mathrm{GeV} / \mathrm{c}$ to be reconstructed with $\mathrm{p}_{\mathrm{T}}>12 \mathrm{GeV} / \mathrm{c}$.

The results of this background calculation are summarized in table $3 \mathrm{~b}$. We find that the corresponding number of decay muon $+\geq 2$ jet events is 0.4 , giving less than 0.10 background events for $\left|\cos \theta_{j_{2}}^{*}\right|<0.8$. In fig. 4 the $\mathrm{E}_{\mathrm{T}}^{\text {out }}$ is plotted versus $\cos \theta_{\mathrm{j}_{2}}^{*}$ for a) the $\pi^{0}+\geq 2$ jet events, and $b$ ) the five muon $+\geq 2$ jet events, and the five $e+\geq 2$ jet events. Of the four muon +2 jet events, one event is most likely a background event of QCD origin since the lowest $E_{T}$ jet $j_{2}$ lies close to the beam axis with $\cos \theta_{\mathrm{j}_{2}}^{*}=0.93$. This event has been removed from the data sample.

\section{BACKGROUNDS DUE TO BEAUTY AND CHARM PAIR PRODUCTION}

Events with the topology of two jets and an isolated large- $\mathrm{p}_{\mathrm{T}}$ lepton can be produced at some level by more conventional processes not containing a t-quark. Of particular relevance is the case in which the prompt lepton is produced by the semileptonic decay of a large- $p_{T} b$-quark or a c-quark. These events ordinarily appear as two jets back-to-back in azimuth with the lepton embedded in one of the two jets and therefore they will not meet our isolation requirements. However, they can simulate the topology of our events provided the lepton is the leading particle (thus suppressing the isolation veto) and another central jet is produced by second-order QCD processes, namely

$$
\begin{aligned}
& \mathrm{gg} \rightarrow \mathrm{gb} \overline{\mathrm{b}}(\mathrm{gc} \overline{\mathrm{c}}), \\
& \mathrm{q} \overline{\mathrm{q}} \rightarrow \mathrm{gb} \overline{\mathrm{b}}(\mathrm{gc \overline {c }}), \\
& \mathrm{qg} \rightarrow \mathrm{qb} \overline{\mathrm{b}}(\mathrm{qc \overline {c }}) .
\end{aligned}
$$

Since the heavy quark cross-sections are expected to be much larger than the $W \rightarrow t \bar{b}$ rate, these backgrounds deserve a careful analysis. So far, QCD predictions for heavy-flavour 
production of large $\mathrm{p}_{\mathrm{T}}$ at the Collider have not been verified by experiment. To this purpose we have selected inclusively all events in which a muon of $\mathrm{p}_{\mathrm{T}}>12 \mathrm{GeV} / \mathrm{c}$ is accompanied by at least one jet of transverse energy $E_{\mathrm{T}}>8 \mathrm{GeV}$, irrespective of the isolation of the muon track. Evidently, this analysis is only possible in the case of muons, since they penetrate the calorimetry and have detectable tracks in the outer muon chambers after all other jet debris have been absorbed. After scanning and excluding events previously identified with $\mathrm{W} \rightarrow \mu v$ and $\mathrm{Z}^{0} \rightarrow \mu^{+} \mu^{-}$decays, we are left with 59 events, mostly containing muons embedded in jets. Background due to pion and kaon decays has been calculated from the inclusive momentum spectrum of charged tracks and found to be $\leq 25 \%$ (and $\leq 10 \%$ for isolated muons). The resulting cross-section for muon inclusive production corrected for detection efficiency is shown in fig. 5. It appears to be in excellent agreement with theoretical calculations [12-15] of large- $\mathrm{p}_{\mathrm{T}}(\mathrm{c} \overline{\mathrm{c}})$ and $(\mathrm{b} \overline{\mathrm{b}})$ production with subsequent semileptonic decay. In order to further verify the associated production nature of the events, we have selected, also inclusively, events with two prompt muons, of either equal or opposite signs and $\mathrm{p}_{\mathrm{T}}>5 \mathrm{GeV} / \mathrm{c}$. The muon transverse momentum spectrum from the resulting 10 events is again in good agreement with the theoretical expectations of ISAJET [11]. QCD calculations of the reactions (1) have been carried out incorporating the requirement that the final state fulfils our selection criteria [ 16]. The background, mainly from $(b \bar{b})$ states with a final hard parton, amounts to at most $1 \%$ of the expected signal from $\mathrm{W} \rightarrow \mathrm{t} \overline{\mathrm{b}}$. Thus, at least within the framework of $\mathrm{QCD}$, the backgrounds due to $(\mathrm{b} \tilde{\mathrm{b}})$ and $(\mathrm{c} \overline{\mathrm{c}})$ production are negligible.

However, in view of the limited experience with these processes, a model-independent determination of this background is highly desirable. Therefore, a more direct method has been employed, which relies empirically on isolation and topology to differentiate between backgrounds and signal. As in the previous section, this analysis is based on the inclusive muon sample. In order to evaluate the effects of the isolation cut, all particles in a cone $\Delta R \leq 1$ around the muon of $\mathrm{p}_{\mathrm{T}}>12 \mathrm{GeV} / \mathrm{c}$ have been neglected. Events with a clean two-jet topology outside this cone have been selected and carefully scanned. In addition to the known, isolated events, 17 other events have been found in which the jet algorithm finds also a jet inside the $\Delta R \leq 1.0$ cone around the muon. These events have all the properties expected from processes (1), namely: i) the higher $\mathrm{E}_{\mathrm{T}}$ jet tends to be back-to-back with the muon in the transverse plane, $\Delta \phi\left(\mu \mathrm{j}_{1}\right) \simeq 180^{\circ}$, and ii) the softer jet is sharply collimated around the incoming beam directions $\left|\cos \theta_{\mathrm{j}_{2}}^{*}\right| \simeq 1$, indicating the gluon bremsstrahlung nature of the softer parton emission. These distributions (shown in fig. 6a) are completely different from the ones for isolated events (fig. $6 \mathrm{~b}$ ), which are somewhat broader in $\Delta \phi\left(\mu \mathrm{j}_{1}\right)$ and flat in $\cos \theta_{\mathrm{j}_{2}}^{*}$. The cut, $\Delta \dot{\phi}\left(\mu \mathrm{j}_{1}\right) \leq 155^{\circ},\left|\cos \theta_{\mathrm{j}_{2}}^{*}\right|<0.8$ removes all 17 non-isolated events and retains $5 / 6$ of the isolated ones. If the isolated events were also of origin (1), both samples should have the same topology, since isolation depends only on the detailed fragmentation of the 'jet' into the muon and other debris. The probability that the two samples have an identical source is $P=3 \times 10^{-4}$, equivalent to a 3.6 st. dev. effect.

Further independent evidence of the different origin of the two samples can be gained by comparing the invariant masses $m\left(\mu v_{\mathrm{T}} \mathrm{j}_{1} \mathrm{j}_{2}\right)$ of isolated and non-isolated events (fig. 7a). Whilst the isolated lepton events (both electrons and muons) cluster around the mass of the $\mathrm{W}$, thus supporting the $\mathrm{W} \rightarrow \mathrm{t} \bar{b}$ hypothesis, the non-isolated muon events are distributed over a broader mass range. Selecting muon events in the mass range $60 \mathrm{GeV} / \mathrm{c}^{2}<\mathrm{m}\left(\mu v_{\mathrm{T}} \mathrm{jj}\right)<100 \mathrm{GeV} / \mathrm{c}^{2}$ and looking at the total energy deposited in a restricted cone $\Delta \mathrm{R} \leq 0.4$, we find predominantly isolated events. For these isolated muons, the additional energy deposition in the cone is consistent with the expected random energy deposition from the underlying event as measured from a clean sample of QCD two-jet events (fig. 7b). Using ISAJET and known fragmentation factors to extrapolate the shape of the background in the region $\Sigma \mathrm{E}_{\mathrm{T}}<2 \mathrm{GeV}$, we find an expected background of 0.15 events, in excellent agreement with theoretical QCD predictions. Therefore we conclude that $b$ and $c$ associated processes cannot be the origin of the observed signal. 


\section{INTERPRETATION OF THE EVENTS}

We will now proceed to examine the physical origin of the six events with a lepton (muon or electron) and two jets. An example of one of these events is shown in fig. 8 . In fig. 9 we show the effective mass of the lepton, two jets, and the transverse component of the neutrino. A very sharp peak can be observed at a value corresponding to the $\mathrm{W}$ mass, once we have allowed for the small shift and broadening arising from the neglect of the longitudinal component of the neutrino momentum [17].

The reconstruction of invariant masses from jets is a novel technique and it deserves some discussion. Jet finding is done in the calorimeters with the standard UAl algorithm which associates both electromagnetic and hadronic cells in $(\eta, \phi)$ space with $\Delta \mathrm{R}=\left(\Delta \eta^{2}+\Delta \phi^{2}\right)^{\frac{1}{2}}<1$. The initiators, which form the core of these jets, must have cells with $\mathrm{E}_{\mathrm{T}} \geq 1.5 \mathrm{GeV}$. Only cells with $|\eta|<2.5$ are used for jet finding. Jets are defined with $\mathrm{E}_{\mathrm{T}}>8 \mathrm{GeV}$ (first jet), whilst other jets within the same event are defined with $\mathrm{E}_{\mathrm{T}}>7 \mathrm{GeV}$. Two corrections have to be introduced to derive the 'true' jet energy: i) some of the jet debris can fall outside the cone of acceptance and therefore energy must be added, and ii) some of the uncorrelated low-energy tracks from the underlying event may be added by the jet-finding algorithm and their average contribution must be subtracted. A very complete Monte Carlo calculation has been set up to take into account these effects, starting from the measured fragmentation functions and including the detector properties. Tables of correction factors and of errors have been generated in this way [18] and used to calculate 'true' energies. It can be seen in fig. 9 that, within these errors, all six events are consistent with a common mass $m_{w}[19]$. Therefore, we conclude that we are observing a new, semileptonic decay of the $W$ particle.

In order to identify the decay mode, we can next evaluate the invariant mass of the lepton, the neutrino, and one of the jets. In fig. 9 , we also show the three-body mass distribution $\mathrm{m}\left(\ell v_{\mathrm{T}} \mathrm{j}_{2}\right)$ obtained by selecting the lower-transverse-energy jet. A sharp peak is observed around $40 \mathrm{GeV} / \mathrm{c}^{2}$. The other solution, based on the other choice of jet, gives a broader spectrum, extending to higher masses (fig. 10). For three events, both choices give consistent mass values. For the other three events, we prefer the low-mass solution since i) the high mass corresponds to decays strongly suppressed by phase space, and ii) Monte Carlo simulation shows that, for $W \rightarrow t \bar{b}$ events, this is the right choice in the majority of cases. Therefore, we conclude that we have observed a new particle state amongst the debris of the $\mathrm{W}$ decays; this state subsequently decays semileptonically. All jets have invariant masses of less than $10 \mathrm{GeV} / \mathrm{c}^{2}$, which sets an upper limit to the mass of the underlying partons [20].

The decay hypothesis $\mathrm{W} \rightarrow \mathrm{t} \overline{\mathrm{b}}$ with $\mathrm{m}_{\mathrm{t}} \simeq 40 \mathrm{GeV} / \mathrm{c}^{2}$ describes all kinematical distributions [21] very well, as shown in fig. 11 . The rate of occurrence of the events, the number of $\mathrm{W} \rightarrow \mathrm{ev}$ decays, and the Monte Carlo determined detection efficiency can be combined in order to evaluate the top semileptonic branching ratio, which is $0.23 \pm 0.09$, to be compared [19] with 0.13 for b-quarks and 0.12 for the lighter, charmed quarks.

Finally, we quote a systematic error of $\pm 10 \mathrm{GeV} / \mathrm{c}^{2}$ in the mass evaluations. This is primarily due to uncertainties in the reconstruction of jets and the determination of the associated parton four-vectors.

In addition to the six lepton +2 jet events we have been discussing, there are three lepton + 3 jet events (table 1c). The rate and topology of these events are not inconsistent with the hypothesis of $(\mathrm{t} t)$ associated production. However, owing to the combinatorial problems and the complexity of the topology, the analysis is more difficult and is still in prcgress. 


\section{CONCLUSIONS}

We observe a clear signal in the channel of an isolated large-transverse-energy lepton plus two or three associated jets. The two-jet signal has an over-all invariant mass clustering around the $\mathrm{W}$ mass, indicating a novel decay of the $\mathrm{W}$. The rates and features of the two-jet events do not satisfy the expectations for charm and beauty decay. They are, however, consistent with the process $\mathrm{W} \rightarrow$ $t \bar{b}$, where $t$ is the sixth 'top' quark of the Cabibbo current. If this is indeed the case, then the mass of the top is bounded between 30 and $50 \mathrm{GeV} / \mathrm{c}^{2}$. We stress that the present uncertainty in the $\left(\ell v_{\mathrm{T}} \mathrm{j}_{2}\right)$ mass is due to the determination of the jet energies, and that more statistics are needed to confirm these conclusions and the true nature of the effect observed.

\section{Acknowledgements}

This result has only been made possible by the magnificent performance of the whole CERN Accelerator Group. We have received enthusiastic support from $\mathrm{H}$. Schopper and I. Butterworth for results emerging from the collider programme.

We are thankful to the technical staff of CERN and of all participating Institutes for their vigorous support of the experiment.

The following funding agencies have contributed to this programme:

Fonds zur Förderung der Wissenschaftlichen Forschung, Austria.

Valtion luonnontieteellinen toimikunta, Finland.

Institut National de Physique Nucléaire et de Physique des Particules and

Institut de Recherche Fondamentale (CEA), France.

Bundesministerium für Forschung und Technologie, Germany.

Istituto Nazionale di Fisica Nucleare, Italy.

Science and Engineering Research Council, United Kingdom.

Department of Energy, USA.

Thanks are also due to the following people who have worked with the Collaboration in the preparations for and data collection on the runs described here: F. Bernasconi, F. Cataneo, R. Del Fabbro, L. Dumps, D. Gregel, J.-J. Malosse, H. Muirhead, G. Salvi, G. Stefanini, R. Wilson, Y.G. Xie and E. Zurfluh.

The help of Mrs. K. Wakley, Mrs. M.-S. Vascotto, Mrs. M. Prola-Tessaur, and Mrs. C. Rigoni in the preparation of this paper is also gratefully acknowledged. 
Table 1a

Event parameters for the isolated electron +2 jet events

\begin{tabular}{|c|c|c|c|c|c|c|c|c|c|c|c|c|c|c|}
\hline \multirow{2}{*}{ Event } & \multicolumn{4}{|c|}{ Electron } & \multirow{2}{*}{$\Delta \mathrm{E}_{\mathrm{m}}$} & \multicolumn{3}{|c|}{ Jet 1} & \multicolumn{4}{|c|}{ Jet 2} & $\left(e v_{T} j_{1} j_{2}\right)$ & \multirow{2}{*}{$m\left(e v_{T} j_{2}\right)$} \\
\hline & Q & $\begin{array}{c}E_{\mathrm{T}} \\
(\mathrm{GeV})\end{array}$ & $\eta$ & $\begin{array}{c}\phi \\
\text { (deg.) }\end{array}$ & & $\begin{array}{c}\mathrm{E}_{\mathrm{T}} \\
(\mathrm{GeV})\end{array}$ & $\begin{array}{c}\phi \\
\text { (deg.) }\end{array}$ & $\eta$ & $\underset{(\mathrm{GeV})}{\mathrm{E}_{\mathrm{T}}}$ & $\begin{array}{c}\phi \\
(\mathrm{deg} .)\end{array}$ & $\eta$ & $\cos \theta^{*}$ & $\left(\mathrm{GeV} / \mathrm{c}^{2}\right)$ & \\
\hline $6301 / 716$ & + & $19.5 \pm 0.8$ & -0.6 & -114 & $6.7 \pm 5.6$ & 14.0 & 29 & 0.1 & 13.9 & 153 & -0.3 & -0.06 & $61 \pm 5$ & $40 \pm 4$ \\
\hline $7443 / 509$ & - & $19.1 \pm 0.7$ & 1.2 & 12 & $4.5 \pm 6.4$ & 23.4 & 134 & 0.2 & 11.1 & -114 & -0.7 & -0.73 & $76 \pm 7$ & $48 \pm 7$ \\
\hline $8578 / 983$ & - & $18.3 \pm 0.7$ & -1.1 & 132 & $5.1 \pm 6.4$ & 22.6 & -19 & 0.5 & 15.5 & -145 & -0.2 & 0.02 & $82 \pm 7$ & $42 \pm 5$ \\
\hline
\end{tabular}

Table 1b

Event parameters for the isolated muon +2 jet events

\begin{tabular}{|c|c|c|c|c|c|c|c|c|c|c|c|c|c|c|}
\hline \multirow{2}{*}{ Event } & \multicolumn{4}{|c|}{ Muon } & \multirow{2}{*}{$\Delta \mathrm{E}_{\mathrm{m}}$} & \multicolumn{3}{|c|}{ Jet 1} & \multicolumn{4}{|c|}{ Jet 2} & $m\left(\mu v_{\mathrm{T}} \mathrm{j}_{1} \mathrm{j}_{2}\right)$ & \multirow{2}{*}{$\mathrm{m}\left(\mu v_{\mathrm{T}} \mathrm{j}_{2}\right)$} \\
\hline & Q & $\underset{(\mathrm{GeV})}{\mathrm{p}_{\mathrm{T}}}$ & $\eta$ & $\begin{array}{c}\phi \\
\text { (deg.) }\end{array}$ & & $\underset{(\mathrm{GeV})}{\mathrm{E}_{\mathrm{T}}}$ & $\begin{array}{c}\phi \\
(\operatorname{deg} .)\end{array}$ & $\eta$ & $\begin{array}{c}\mathrm{E}_{\mathrm{T}} \\
(\mathrm{GeV})\end{array}$ & $\begin{array}{c}\phi \\
\text { (deg.) }\end{array}$ & $\eta$ & $\cos \theta^{*}$ & $\left(\mathrm{GeV} / \mathrm{c}^{2}\right)$ & \\
\hline $6639 / 118$ & + & $16.0 \pm 3.4$ & 0.9 & 103 & $5.3 \pm 7.0$ & 30.0 & -70 & -0.4 & 11.1 & 23 & -1.0 & -0.76 & $\begin{array}{c}79-7 \\
+10\end{array}$ & $\begin{array}{r}41-6 \\
+11\end{array}$ \\
\hline $7501 / 117$ & - & $13.5 \pm 1.0$ & -0.3 & 112 & $7.5 \pm 7.0$ & 22.9 & -41 & -0.8 & 20.2 & -179 & -1.0 & -0.30 & $75 \pm 7$ & $43 \pm 9$ \\
\hline $7935 / 232$ & - & $16.2 \pm 3.3$ & 0.4 & 65 & $\begin{array}{r}5.8+7.0 \\
-6.0\end{array}$ & 25.1 & -140 & 1.7 & 11.3 & -13 & 1.0 & -0.25 & 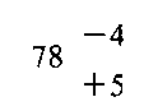 & $37 \pm 9$ \\
\hline
\end{tabular}

Table 1c

Event parameters for the isolated lepton +3 jet events

\begin{tabular}{|c|c|c|c|c|c|c|c|c|c|c|c|c|c|c|}
\hline \multirow{2}{*}{ Event } & \multirow{2}{*}{ Type } & \multicolumn{3}{|c|}{ Lepton } & \multirow{2}{*}{$\Delta \mathrm{E}_{\mathrm{m}}$} & \multicolumn{3}{|c|}{ Jet 1} & \multicolumn{3}{|c|}{ Jet 2} & \multicolumn{3}{|c|}{ Jet 3} \\
\hline & & $\mathrm{E}_{\mathrm{T}}$ & $\eta$ & $\phi$ & & $\mathrm{E}_{\mathrm{T}}$ & $\phi$ & $\eta$ & $\mathrm{E}_{\mathrm{T}}$ & $\phi$ & $\eta$ & $\mathrm{E}_{\mathrm{T}}$ & $\phi$ & $\eta$ \\
\hline ' & & & & & & & & & & & & & & \\
\hline $7700 / 487$ & $\mu^{-}$ & $21.4 \pm 2.6$ & 1.7 & -12 & $2.2 \pm 7.0$ & 22.9 & -161 & 1.3 & 12.0 & 61 & 1.8 & 9.5 & 31 & 0.3 \\
\hline $5069 / 192$ & $\mathrm{e}^{+}$ & $15.0 \pm 0.7$ & 0.7 & -41 & $26.7 \pm 5.7$ & 19.5 & -150 & -0.7 & 14.1 & 32 & -0.5 & 13.0 & -149 & 0.8 \\
\hline $6899 / 804$ & $e^{-}$ & $18.0 \pm 0.8$ & -0.3 & -58 & $9.7 \pm 5.7$ & 16.9 & 83 & 0.6 & 8.8 & -121 & -0.1 & 8.5 & 169 & -0.7 \\
\hline
\end{tabular}


Table 2

The number of events in the two regions of the $\mathrm{E}_{\mathrm{T}}^{\text {out }}$ versus $\cos \theta_{\mathrm{j}_{2}}^{*}$ plane (see text)

\begin{tabular}{lcc}
\hline \multicolumn{1}{c}{ Region } & $\pi^{0}$ & $\mathrm{e}$ \\
\hline$\pi^{0} /$ electron $+\geq 2$ jets $(\mathrm{RI}+\mathrm{RII})$ & 769 & 5 \\
$\mathrm{RI}=\left\{\mathrm{E}_{\mathrm{T}}^{\text {out }}>8 \mathrm{GeV},\left|\cos \theta_{\mathrm{J}}^{*}\right|<0.73\right\}$ & 162 & 5 \\
$\mathrm{RII}=1-\mathrm{RI}$ & 607 & 0 \\
\hline
\end{tabular}


Expected background to $e+\geq 1$ jet events arising from charged pions faking an isolated electron with $\mathrm{E}_{\mathrm{T}}>15 \mathrm{GeV}$. Both the normal trigger data sample and a low threshold $\left(5 \mathrm{nb}^{-1}\right)$ trigger data sample give consistent results.

\begin{tabular}{llll}
\hline \multirow{2}{*}{ Event sample } & Channel & \multicolumn{2}{c}{ Expected background } \\
\cline { 2 - 4 } & & Isolated $^{\text {a) }}$ & Super-isolated $^{\mathrm{b})}$ \\
\hline Normal trigger & $\pi^{ \pm}+1$ jet & $\begin{array}{l}0.7 \pm 0.2 \\
0.36 \pm 0.14\end{array}$ & $\begin{array}{l}0.3 \pm 0.1 \\
0.10 \pm 0.04\end{array}$ \\
\hline \multirow{2}{*}{$\pi^{ \pm}+2$ jets } & $\pi^{ \pm}+1$ jet & $1.1 \pm 0.3$ & $0.65 \pm 0.25$ \\
Low-threshold $\left(5 \mathrm{nb}^{-1}\right)$ & $\pi^{ \pm}+2$ jets & $0.3 \pm 0.2$ & $0.06 \pm 0.04$ \\
\hline
\end{tabular}

a) Isolation criteria in a cone $\Delta \mathrm{R} \leq 0.4 ; \Sigma \mathrm{E}_{\mathrm{T}}<1 \mathrm{GeV}$, and $\Sigma \mathrm{p}_{\mathrm{T}}<\mathrm{IGeV} / \mathrm{c}$ (see text).

b) Super-isolation criteria, demanding the additional isolation requirements in a cone $\Delta \mathrm{R} \leq 0.7 ; \Sigma \mathrm{E}_{\mathrm{T}}<2 \mathrm{GeV}$, and $\Sigma \mathrm{p}_{\mathrm{T}}<1.5 \mathrm{GeV} / \mathrm{c}$. The candidate $\mathrm{e}+\geq 2$ jet events satisfy these criteria.

Table $3 b$

Expected background to $\mu+\geq 1$ jet events arising from the decay of charged pions and kaons in the central detector volume. The low-threshold trigger $\left(5 \mathrm{nb}^{-1}\right)$ data sample has been used. There is a systematic uncertainty of a factor of 2 on the normalization of these estimates.

\begin{tabular}{lccc}
\hline \multicolumn{1}{c}{ Cuts } & Events & \multicolumn{2}{c}{ Background estimate } \\
\cline { 3 - 4 } & & No isolation & Isolated $^{\mathrm{a})}$ \\
\hline $\begin{array}{l}\mathrm{p}_{\mathrm{T}}^{\mu}>12 \mathrm{GeV} / \mathrm{c} ; \\
\geq 1 \text { jet with } \mathrm{E}_{\mathrm{T}}>8 \mathrm{GeV} \\
\text { and }|\eta|<2.5\end{array}$ & 6 & 9.0 & 0.9 \\
\hline $\begin{array}{l}\Delta \mathrm{R}(\mu, \text { jet })>1^{\text {b) }} \\
\geq 2 \text { jets with } \mathrm{E}_{\mathrm{T}}>7 \mathrm{GeV} \\
\text { and }|\eta|<2.5\end{array}$ & 4 & 3.8 & 0.4 \\
\hline $\begin{array}{l}\text { Exactly } 2 \text { jets } \\
\text { with } \mathrm{E}_{\mathrm{T}}>7 \mathrm{GeV}\end{array}$ & 3 & 2.4 & 0.2 \\
\hline \begin{tabular}{l}
$\left|\cos \theta_{\mathrm{j}_{2}}^{*}\right|<0.8$ \\
\hline
\end{tabular} & 3 & 1.5 & 0.1 \\
\hline
\end{tabular}

a) Isolation in a cone $\Delta R \leq 0.4$.

b) Distance $\Delta \mathrm{R}$ between $\mu$ and nearest jet with $\mathrm{E}_{\mathrm{T}}>7 \mathrm{GeV}$. 


\section{REFERENCES AND FOOTNOTES}

[1] G. Arnison et al., Phys. Lett. 122B (1983) 103.

G. Arnison et al., Phys. Lett. 129B (1983) 273.

C. Rubbia, Experimental observation of events with large missing transverse energy accompanied by a jet or a photon(s) in p $\bar{p}$ collisions at the $\sqrt{s}=540 \mathrm{GeV}$, talk given at the $4^{\text {th }}$ Topical Workshop on Proton-Antiproton Collider Physics, Berne, 1984.

[2] G. Arnison et al., Phys. Lett. 134B (1984) 469.

[3] UA1 Collaboration, Hadronic jet activity associated with intermediate vector boson production at the SPS Collider, presented by S. Geer at the $19^{\text {th }}$ Rencontre de Moriond, La Plagne, 1984. The contribution from leptonic $W$ decays to the lepton plus two jet channel is negligible after requiring a missing transverse energy of less than $15 \mathrm{GeV}$ and two central jets with transverse energy greater than $7 \mathrm{GeV}$.

[4] TASSO Collaboration, M. Althoff et al., Phys. Lett. 138B(1984) 441.

S. Yamada, Proc. 1983 Intern. Symp. on Lepton and Photon Interactions at High Energies (Cornell, 1983); and DESY report 83-100(1983).

Mark J Collaboration, B. Adeva et al., Phys. Rep. 109(1984) 1.

S.L. Wu, Phys. Rep. 107 (1984) 1.

[5] G. Arnison et al., Phys. Lett. 139B (1984) 115.

The scalar missing energy $\Delta \mathrm{E}_{\mathrm{m}}$ is defined as the apparent momentum imbalance in the transverse plane, obtained by adding energy depositions vectorially in all calorimeter cells. Since the calorimetry covers essentially a $4 \pi$ solid angle, this quantity reflects the transverse component of the momentum of the emitted neutrino(s).

[6] The invariant mass $m_{t}$ reflects the 'bare' quark mass. Small changes are expected for physical top particles, in which the top quark is bound to 'ordinary', much lighter quarks. Within the present accuracy, this effect can be neglected.

[7] M. Barranco Luque et al., Nucl. Instrum. Methods 176(1980) 75.

M. Calvetti et al., Nucl. Instrum. Methods 176(1980) 255.

M. Calvetti et al., IEEE Trans. Nucl. Sci. NS-30(1983) 71.

M. Corden et al., Phys. Scr. 25 (1982) 5 and 11.

K. Eggert et al., Nucl. Instrum. Methods 176 (1980) 217.

The UA1 Collaboration is preparing a comprehensive report on the detector, to be published in Nucl. Instrum. Methods (NIM).

[8] M. Della Negra et al., Expected rate of background to the electron sample from conversions, CERN UA1-TN/84-76(1984).

[9] E. Eisenhandler et al., CERN UA 1 -TN/84-64 (1984).

$\chi_{\mathrm{R}}^{2}$ measures the goodness of fit to the electron hypothesis. This parameter has bee $\mathrm{n}$ developed from a study of electron test-beam data making full use of the longitudinal profile of the shower measured in the four samplings of the electromagnetic calorimeter, and taking into account the electron energy and angle of incidence.

[10] This effect is well understood according to QCD since the third jet is mostly due to gluon bremsstrahlung from the incoming partons. Experimental verification of this rather general property has been observed at the collider both for QCD jets and for $\mathrm{W}+$ jet events. For more details, see ref. [3].

[11] M. N. Minard, B. Sadoulet and I. Wingerter, Background study to e + jet events, CERN UA1-TN/84-77(1984).

[12] R. Horgan and M. Jacob, Nucl. Phys. B238 (1984) 221.

[13] F. Halzen and D.M. Scott, Phys. Lett. 129B (1983) 341. 
[14] R. Kinnunen, private communication, and Ph.D. thesis, Univ. Helsinki, 1984 (to be published).

[15] F.E. Paige and S.D. Protopopescu, ISAJET program, BNL 29777 (1981).

B. Humpert, Phys. Lett. 85B (1979) 293.

[16] I. Schmitt, L.M. Sehgal, H. Thode and P.M. Zerwas, Simulation of t-quark production by a hard three-parton final state in $\bar{p} \overline{\mathrm{c}}$ collisions, Univ. Aachen preprint PITHA 83/26 (1983).

L.M. Sehgal and P.M. Zerwas, Univ. Aachen preprint PITHA 83/10 (1983), Nucl. Phys. B, in press.

V. Barger, A.D. Martin and R.J.N. Phillips, Phys. Lett. 125B (1983) 343; Phys. Rev. D28 (1983) 145.

R.M. Godbole, S. Pakvasa and D.P. Roy, Phys. Rev. Lett. 50(1983) 1539.

F. Halzen and D.M. Scott, Phys. Lett. 129B (1983) 341.

K. Hagiwara and W.F. Long, Madison report MAD/PH/117 (1983).

R. Horgan and M. Jacob, CERN preprint Ref. TH.3682 (1983).

J.H. Kühn, Fermilab preprint PUB-83/79-THY (1983).

V. Barger, H. Baer, A.D. Martin and R.J.N. Phillips, Madison report MAD/PH/133 (1983).

R. Odorico, CERN preprint Ref. TH.3678 (1983).

[17] R. Kinnunen, see ref. [14].

[18] M. Della Negra et al., CERN UA 1-TN/84-43 (1984).

M. Della Negra and P. Ghez, CERN UA 1-TN/84-15 (1984).

Note that ISAJET [15] gives systematically different results by as much as $10-20 \%$ on the jet energies.

[19] Particle Data Group, Review of particle properties, Rev. Mod. Phys. 56 (1984).

[20] This strongly suggests a decay into $u, d, c, b$ quarks, since the quark spectrum below $10 \mathrm{GeV} / \mathrm{c}^{2}$ is well known and fully explored by $\mathrm{e}^{+} \mathrm{e}^{-}$experiments.

[21] V. Barger et al., CERN preprint Ref. TH.3972 (1984), submitted to Physics Letters. 
Fig. 1 : Identification of $\pi^{0}$ conversions. a) The minimum distance $\mathrm{D}$ (at the point where the tracks are parallel) between the energetic electron candidate and the nearest track in the drift plane of the central detector is shown normalized by the error on this quantity. When the unreconstructed partners recognized by visual scanning are included, a cut at $3 \sigma$ removes 43 conversions. b) The rate of identified conversions shown as a function of the transverse momentum of the low- $\mathrm{p}_{\mathrm{T}}$ partner, compared with the expected rate [8]. The loss at higher values of $\mathrm{p}_{\mathrm{T}}$ reflects the isolation criteria imposed on the energetic electron.

Fig. 2 : Electron isolation. The electron quality parameter $\chi_{R}^{2}[9]$ is shown as a function of the energy accompanying the electron candidate in a cone of $\Delta R \leq 0.4$ around the track. This is shown for a) $\mathrm{W} \rightarrow \mathrm{e}^{ \pm} v_{\mathrm{e}}$ events, b) $\mathrm{e}^{ \pm}+$single jet events, and c) $\mathrm{e}^{ \pm}+\geq 2$ jet events.

Fig. 3 : Electron quality. The quality of the electrons in the e $+\geq 2$ jet sample (shaded) is compared with the control sample of electrons from $\mathrm{W}^{ \pm} \rightarrow \mathrm{e}^{ \pm} v_{\mathrm{e}}$ decays. The quality variables shown are: a) the matching between the momentum measurement and the calorimeter energy deposition; b) the quality of the matching between the track direction measured in the central detector and the direction measured by pulse division in each of the four segments of the e.m. calorimeter; c) an over-all quality parameter $\chi_{\mathrm{R}}^{2}$ [9] measuring the electromagnetic shape of the longitudinal shower profile and the pulse sharing between the different calorimeter samples; and d) the energy deposited in the hadron calorimeter behind the electromagnetic shower.

Fig. 4 : a) Measured shape of the expected QCD background extracted from $\pi^{0}+\geq 2$ jet events. The transverse energy component of the isolated $\pi^{0}$ perpendicular to the plane formed by the $\mathrm{p} \overline{\mathrm{p}}$ axis and the highest- $\mathrm{E}_{\mathrm{T}}$ jet, $\mathrm{E}_{\mathrm{T}}^{\text {out }}$ is plotted as a function of $\cos \theta_{\mathrm{j}_{2}}^{*}$. The angle $\theta_{\mathrm{J}_{2}}^{*}$ is between the average beam axis in the three-body rest frame and the lowest- $E_{\mathrm{T}}$ jet $\left(\mathrm{j}_{2}\right)$. b) As above but for the isolated electron $+\geq 2$ jet events (open circles) and the isolated muon $+\geq 2$ jet events (full circles).

Fig. $5 \quad$ : Measured inclusive muon $\mathrm{p}_{\mathrm{T}}$ spectrum (solid squares) with no isolation cuts. The upper curves are theoretical predictions of the inclusive muon rate from $b$ - and c-quarks: Horgan and Jacob [12] (dashed), Halzen and Scott [13] (dot-dashed), and Kinnunen [14] (solid). Also plotted is the muon $\mathrm{p}_{\mathrm{T}}$ spectrum from dimuon events (solid circles, two entries per event). The lower curve (dotted) is a prediction, using ISAJET [15], of the dimuon rate from $b$ - and c-quark decay assuming a total cross-section consistent with the inclusive single muon rate.

Fig. 6 : Lepton +2 jet event shape for a) the non-isolated muon sample for which the lowest $\mathrm{E}_{\mathrm{T}}$ jet is central $\left(\left|\cos \theta_{\mathrm{j}}^{*}\right|<0.8\right)$, and $\mathrm{b}$ ) the isolated electron and isolated muon samples. The angle in the transverse plane between the lepton and the highest $E_{\mathrm{T}}$ jet, $\Delta \phi\left(\ell \mathrm{j}_{1}\right)$, is shown as a function of $\cos \theta_{\mathrm{j}_{2}}^{*}$ (see fig. 4). The curves show the expectation [3] for a) QCD background events from process (1), and b) $W \rightarrow t \bar{b}$ events with a top-quark mass $\mathrm{m}_{\mathrm{t}}=40 \mathrm{GeV} / \mathrm{c}^{2}$.

Fig. 7 : Four-body mass distribution for isolated muon +2 jet events and non-isolated muon (muons accompanied by a hadronic jet) +2 jet events. The mass of the $\left(\mu v_{\mathrm{r}} \mathrm{j}_{1} \mathrm{j}_{2}\right)$ system is plotted as a function of the muon isolation parameter $\Sigma \mathrm{E}_{\mathrm{T}}$, the additional energy deposited is a cone $\Delta R=0.4$ around the muon. a) The isolated muon (shaded) events show a peak at the $\mathrm{W}$ mass. The distribution for the isolated events agrees with that expected for genuinely isolated muons, the $\Sigma E_{T}$ arising from a random 
contribution from the underlying event (solid curve). The $\Sigma \mathrm{E}_{\mathrm{T}}$ distribution for the non-isolated events agrees with an ISAJET prediction for the background from process (1) (broken curve). b) The $\Sigma \mathrm{E}_{\mathrm{T}}$ distribution is shown for those events lying in the region of the $W$ mass in the $m\left(\mu v_{\mathrm{T}} \mathrm{j}_{1} \mathrm{j}_{2}\right)$ distribution. These events are predominantly isolated (solid curve) with only a small contamination from QCD background events (broken curve).

Fig. $8:$ Graphic display of calorimeter cells $\left(\mathrm{E}_{\mathrm{T}}>2 \mathrm{GeV}\right)$ and charged tracks $\left(\mathrm{p}_{\mathrm{T}}>1.5\right.$ $\mathrm{GeV}$ ) observed in the UA 1 detector for event $7443 / 509$, a $W^{-} \rightarrow$ tb candidate. a) General view, and $b$ ) view looking along the beam direction. The decay products $(b, \bar{b}, \mathrm{e}$ - and $\bar{v}$ ) are labelled.

Fig. 9 : Four-body versus three-body mass distribution for the $\operatorname{six} \mathrm{W} \rightarrow \mathrm{t} \overline{\mathrm{b}}$ candidate events. The effective mass of the lepton, the lower- $E_{\mathrm{T}}$ jet, and of the transverse component of the neutrino is plotted against the mass of the lepton, two-jet, transverse neutrino system. The four-body mass peaks at the $\mathrm{W}$ mass. The three-body mass clusters around a common value of $\sim 40 \mathrm{GeV} / \mathrm{c}^{2}$. The curves show the expected [17] distributions, taking into account the experimental resolution. Allowance should be made for a systematic error arising from uncertainties in the jet reconstruction $\left( \pm 10 \mathrm{GeV} / \mathrm{c}^{2}\right)$.

Fig. 10 : The two solutions for the three-body mass distributions $m\left(\ell v_{\mathrm{T}} \mathrm{j}\right)$. The solution using the lower- $E_{\mathrm{T}}$ jet, $\mathrm{m}\left(\ell_{\mathrm{r}_{\mathrm{T}}} \mathrm{j}_{2}\right)$, gives a distribution consistent with a common value of $\sim 40 \mathrm{GeV} / \mathrm{c}^{2}$. The other solution using the higher- $\mathrm{E}_{\mathrm{T}}$ jet gives a broader distribution extending to higher masses.

Fig. 11 : Kinematic distributions for the six $\mathrm{W} \rightarrow \mathrm{t} \overline{\mathrm{b}}$ candidates, compared with theoretical expectations [21] for a top mass $m_{t}=40 \mathrm{GeV} / \mathrm{c}^{2}$. a) Mass distributions for i) the lepton two-jet system $m\left(j_{1} j_{2} \ell\right)$; ii) the lepton highest- $E_{r}$ jet system $m\left(j_{1} \ell\right)$; and iii) the lepton lowest- $\mathrm{E}_{\mathrm{T}}$ jet system $\mathrm{m}\left(\mathrm{j}_{2} \ell\right)$. b) Transverse mass distributions defined in ref. [21]:

i) $m_{T}(1) ; m_{T}^{2}(1) \equiv m_{W}^{2}+m_{b}^{2}-2 m_{W}\left(m_{b}^{2}+\bar{b}_{T}^{2}\right)^{\frac{1}{2}}$, where $\bar{b}_{\mathrm{T}}$ is the transverse momentum of the highest- $\mathrm{E}_{\mathrm{T}}$ jet.

ii) $\mathrm{m}_{\mathrm{T}}(2) \equiv \mathrm{m}_{\mathrm{T}}(\mathrm{b} \ell, v)$, where $\mathrm{m}_{\mathrm{T}}^{2}(\mathrm{c} v) \equiv\left(\mathrm{c}_{\mathrm{T}}^{0}+v_{\mathrm{T}}\right)^{2}-\left(\overrightarrow{\mathrm{c}}_{\mathrm{T}}+\vec{v}_{\mathrm{T}}\right)^{2}$ and $\mathrm{c}_{\mathrm{T}}^{0}=\left(\overrightarrow{\mathrm{c}}_{\mathrm{T}}^{2}+\mathrm{m}_{\mathrm{c}}^{2}\right)^{\frac{1}{2}}$.

iii) $\mathrm{m}_{\mathrm{T}}(3) \equiv \mathrm{m}_{\mathrm{T}}(\overline{\mathrm{b}} \mathrm{b} \ell ; v)$. 


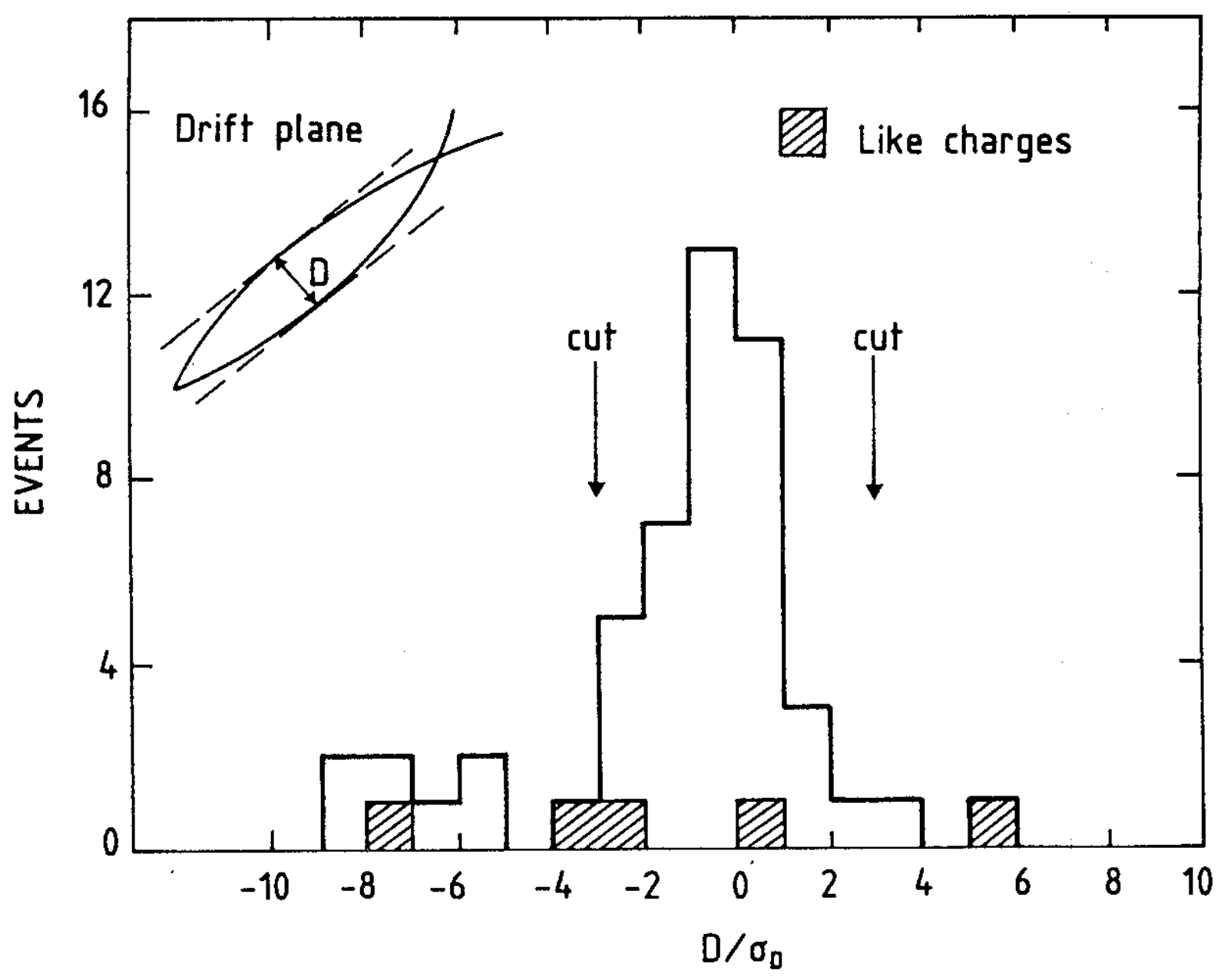

Fig. 1a 


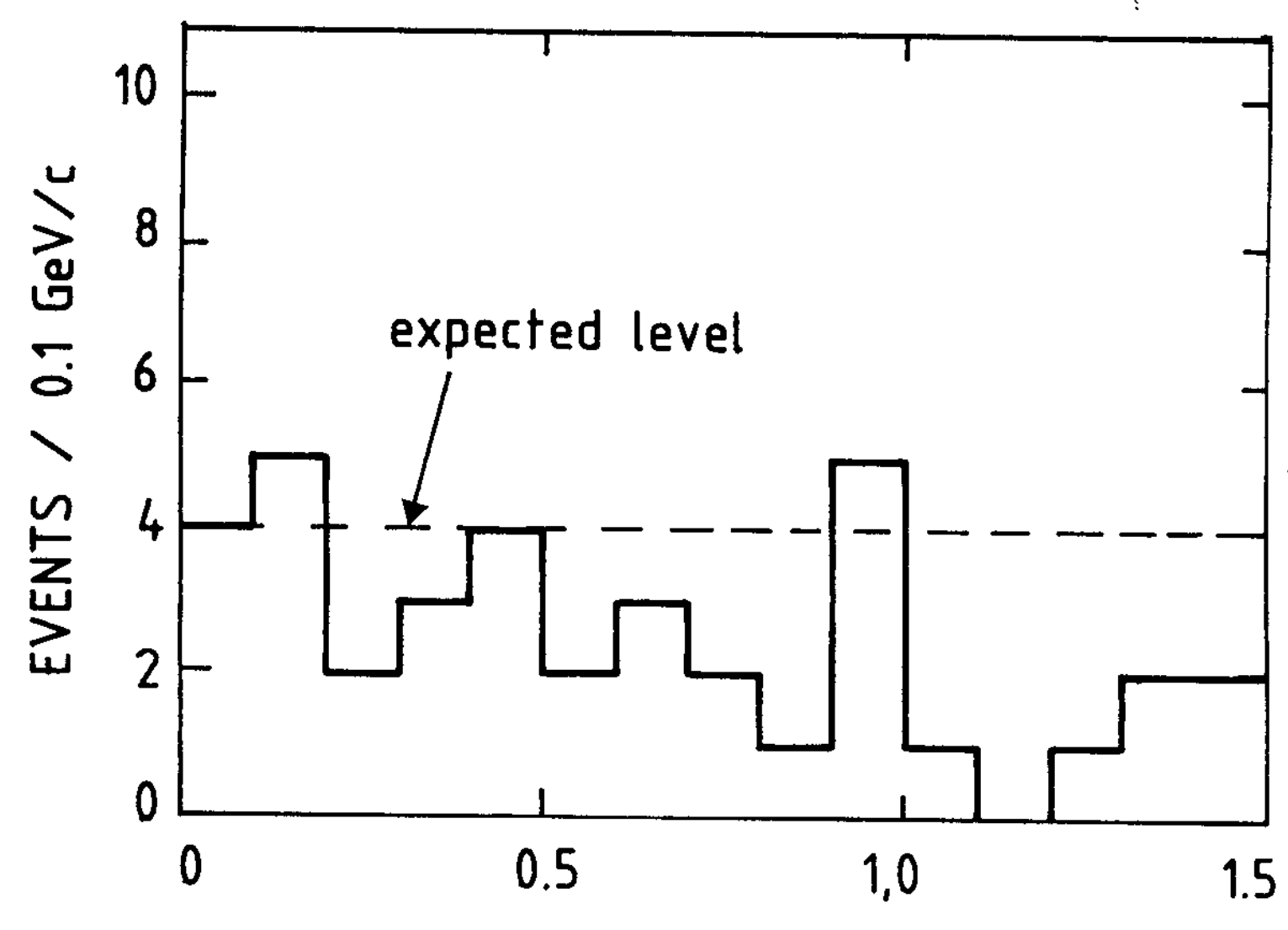

Transverse momentum of second electron $(\mathrm{GeV} / \mathrm{c})$

Fig. Ib 
$W \rightarrow$ eV EVENTS

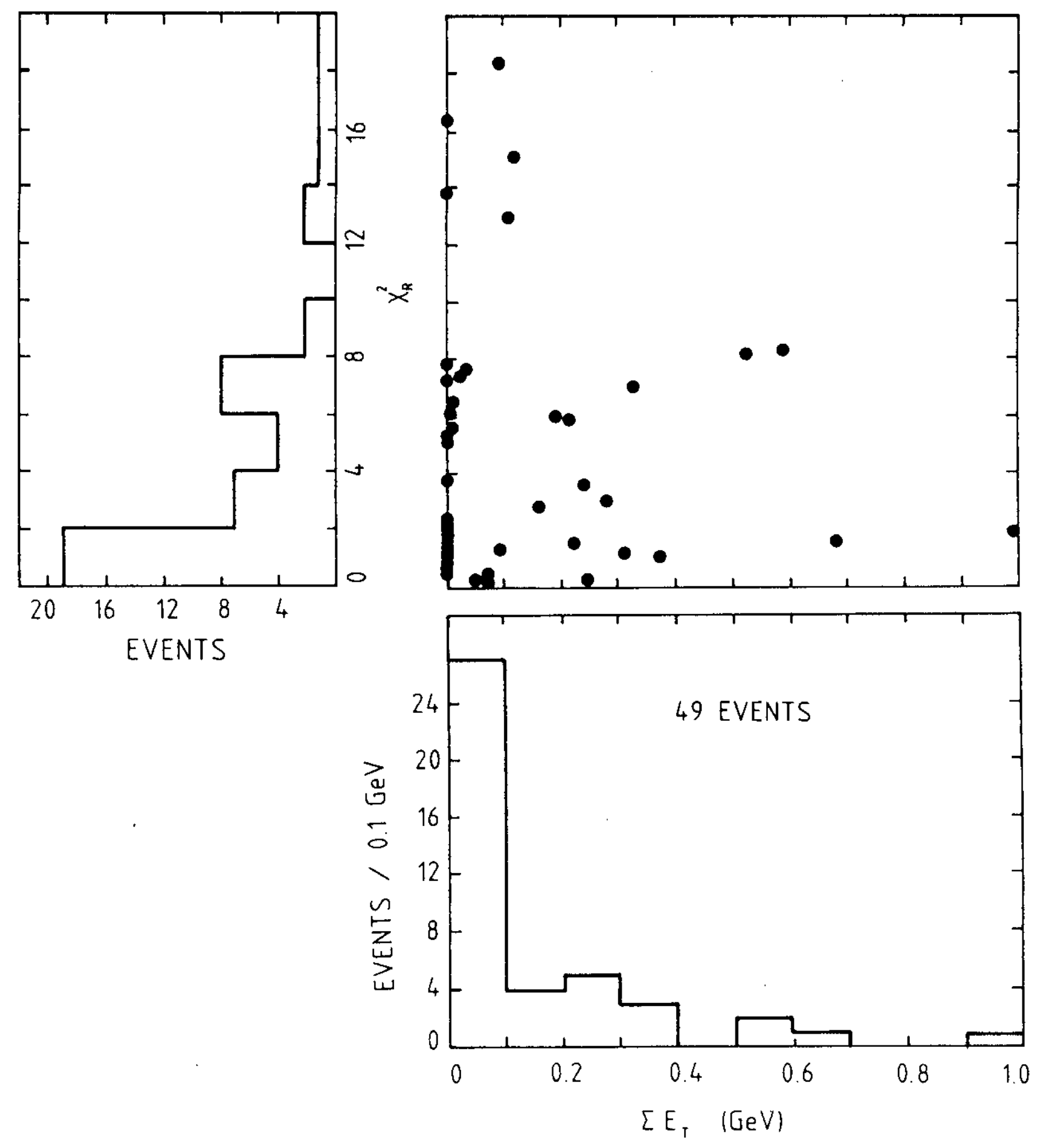

Fig. $2 a$ 
$(e+1$ jet) EVENTS

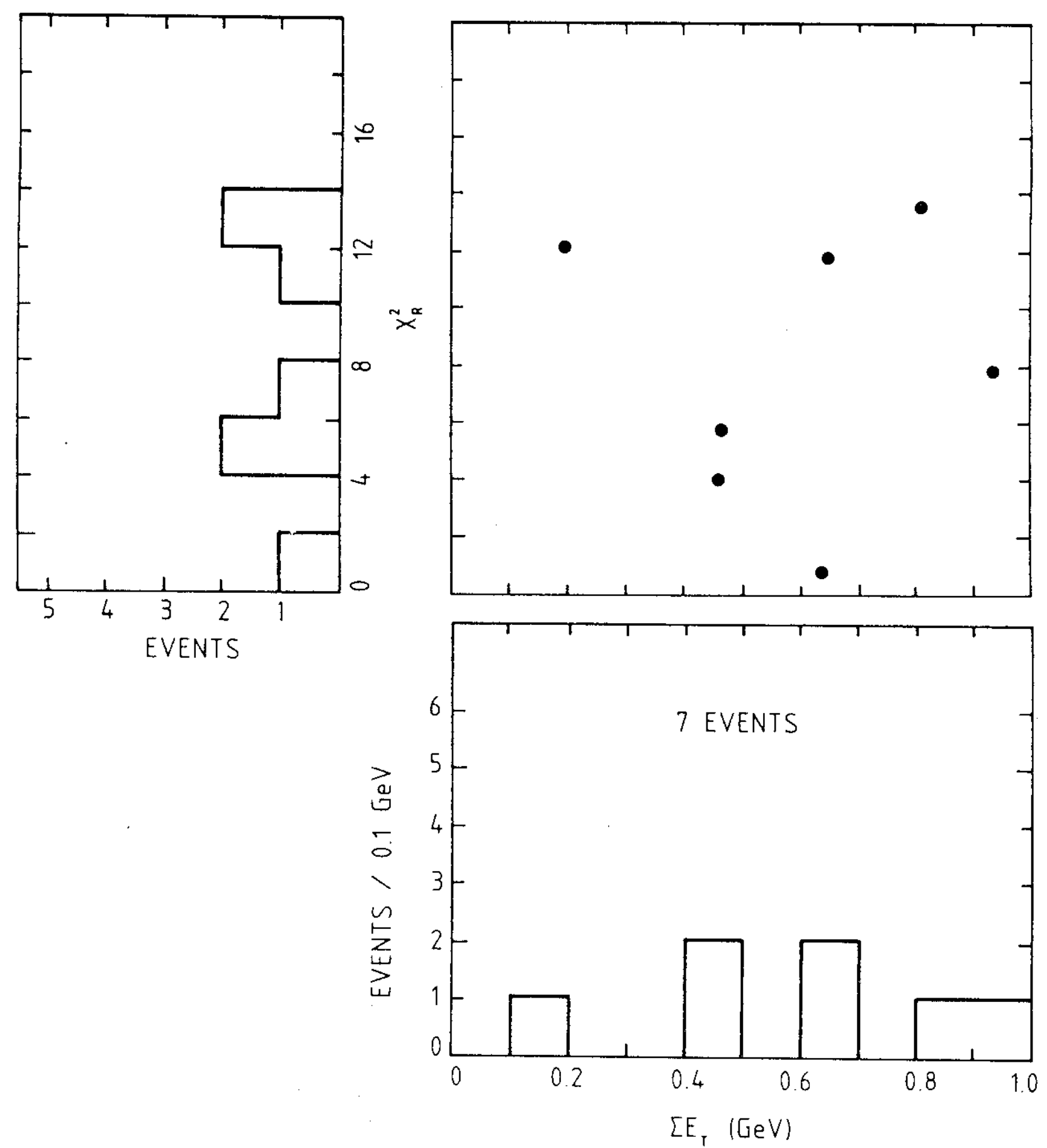

Fig. 2b 
- (e+2 jet) Events

o (e +3 jet) Events

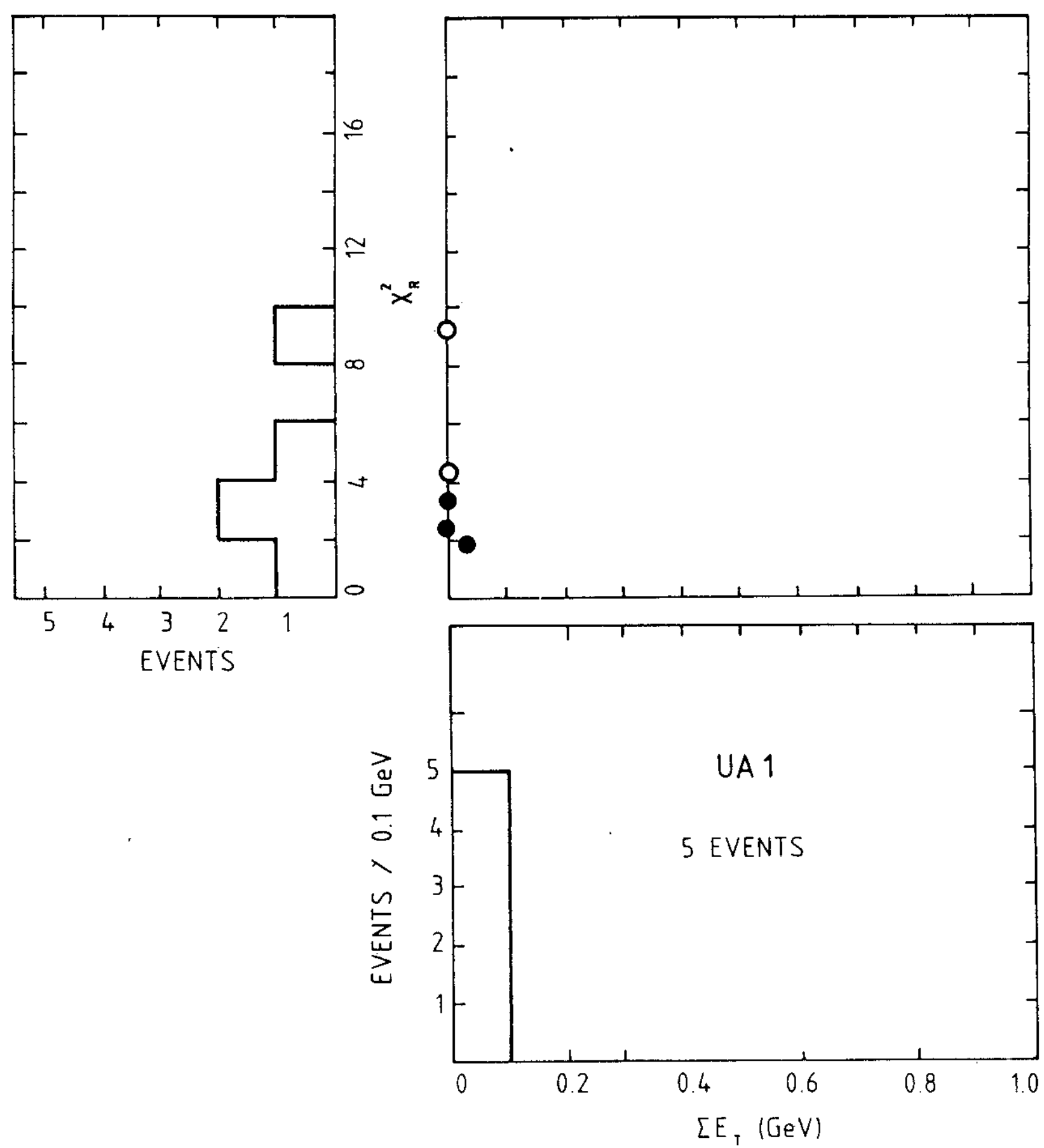

Fig. 2c 


\section{$\square \mathrm{W} \rightarrow$ eV (49 EVENTS) \\ ए $+\geqslant 2$ JETS (5 EVENTS)}
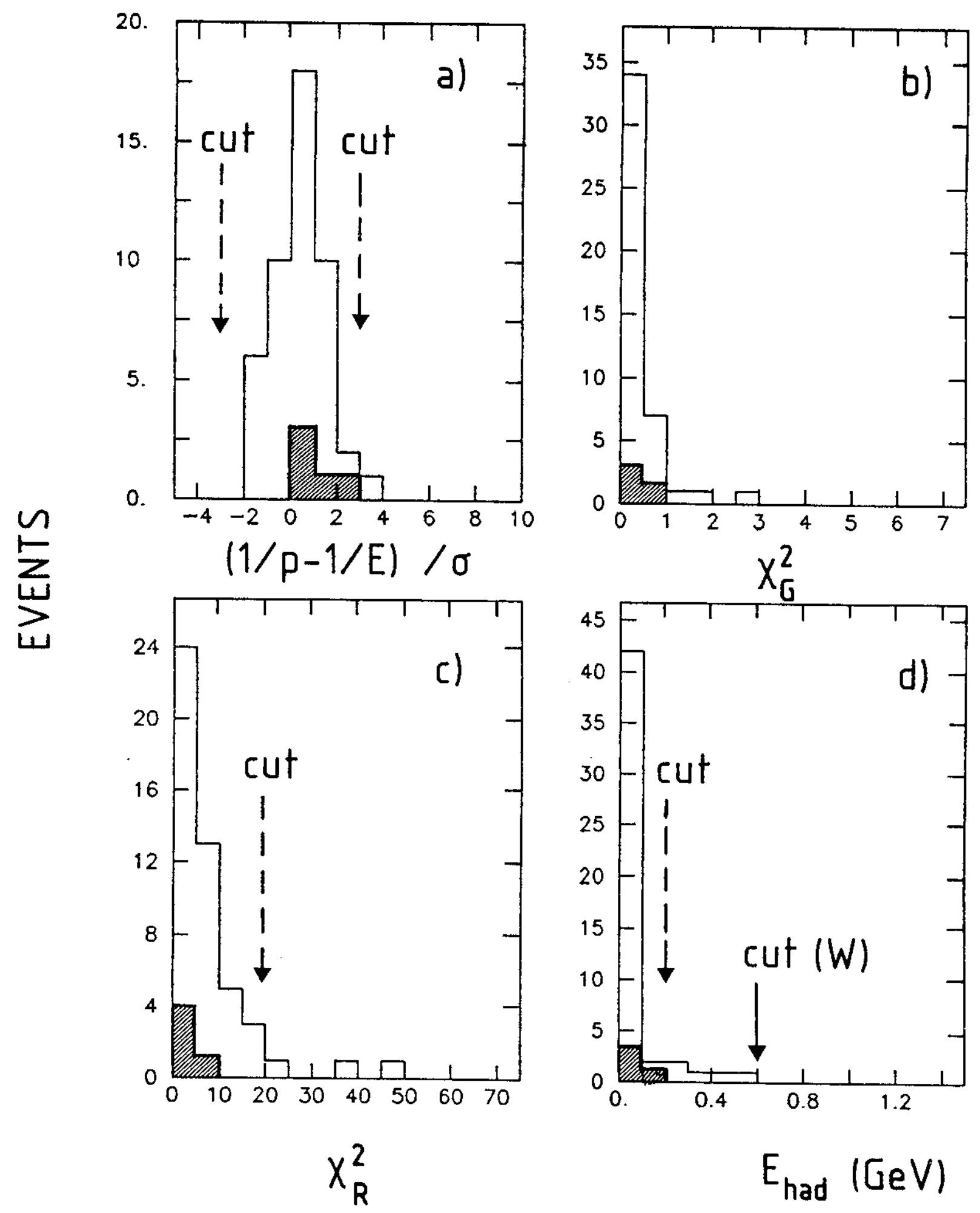

$E_{\text {had }}(\mathrm{GeV})$

Fig. 3 


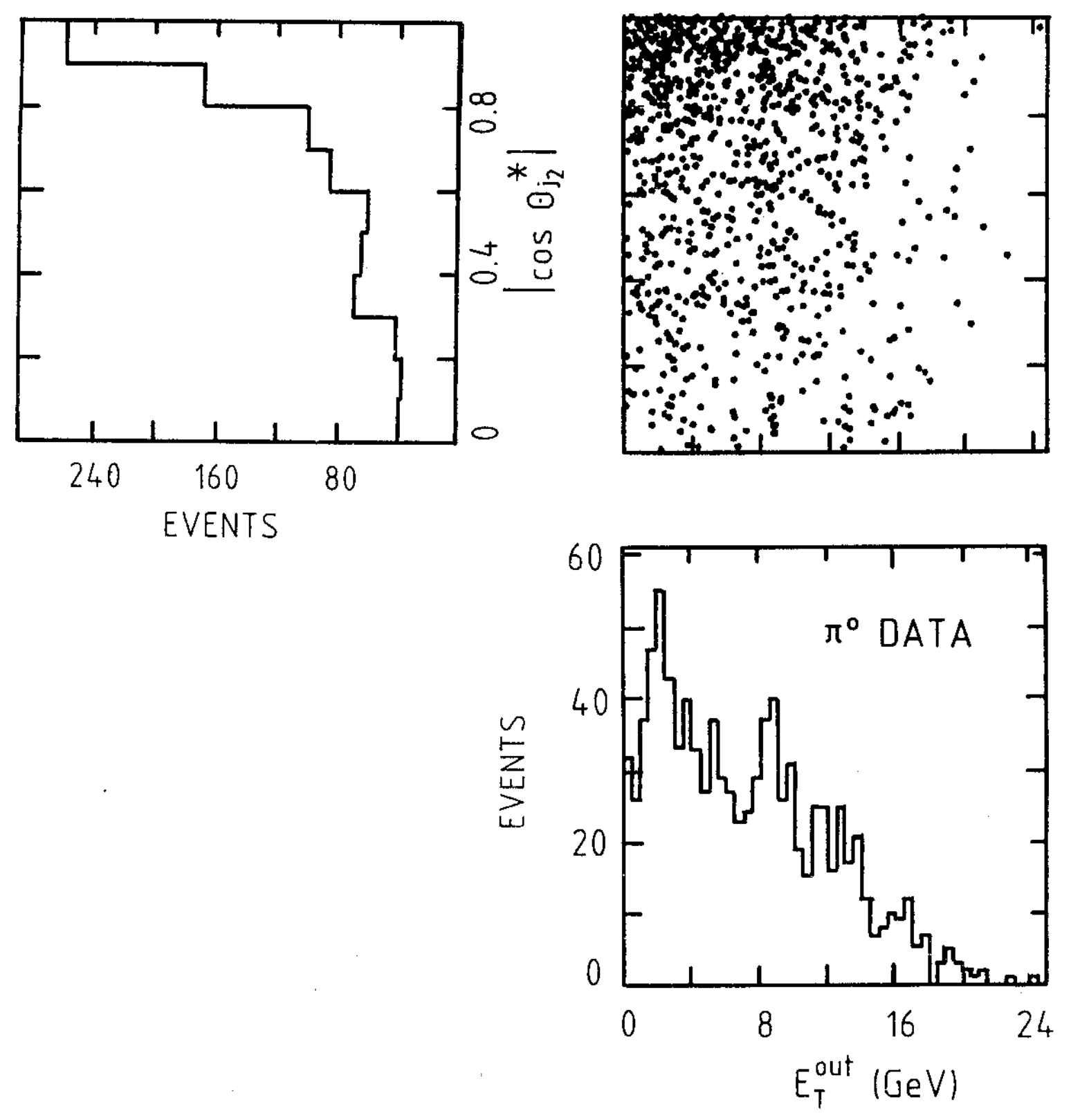

Fig. $4 a$ 


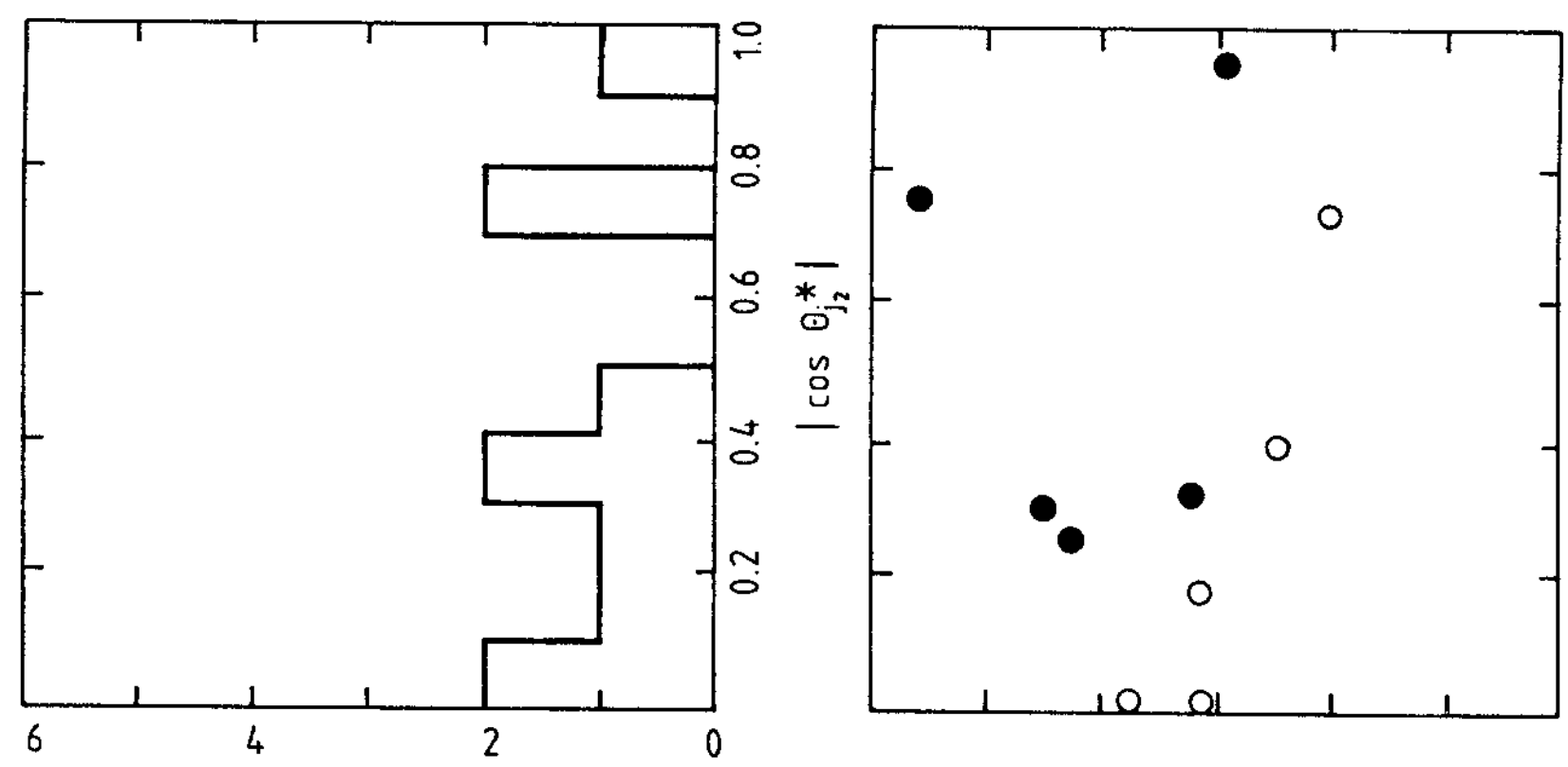

EVENTS

$\mathrm{O}$ e $t \geqslant 2$ jets

- $\mu+\geqslant 2$ jets

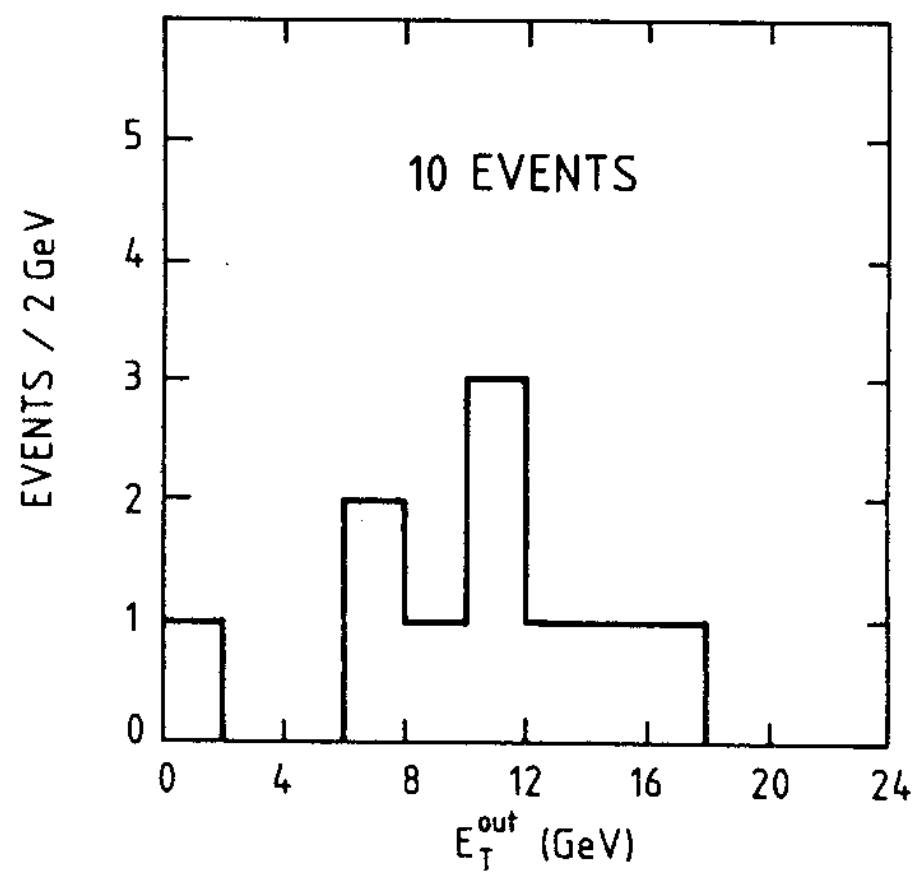

Fig. 4b 


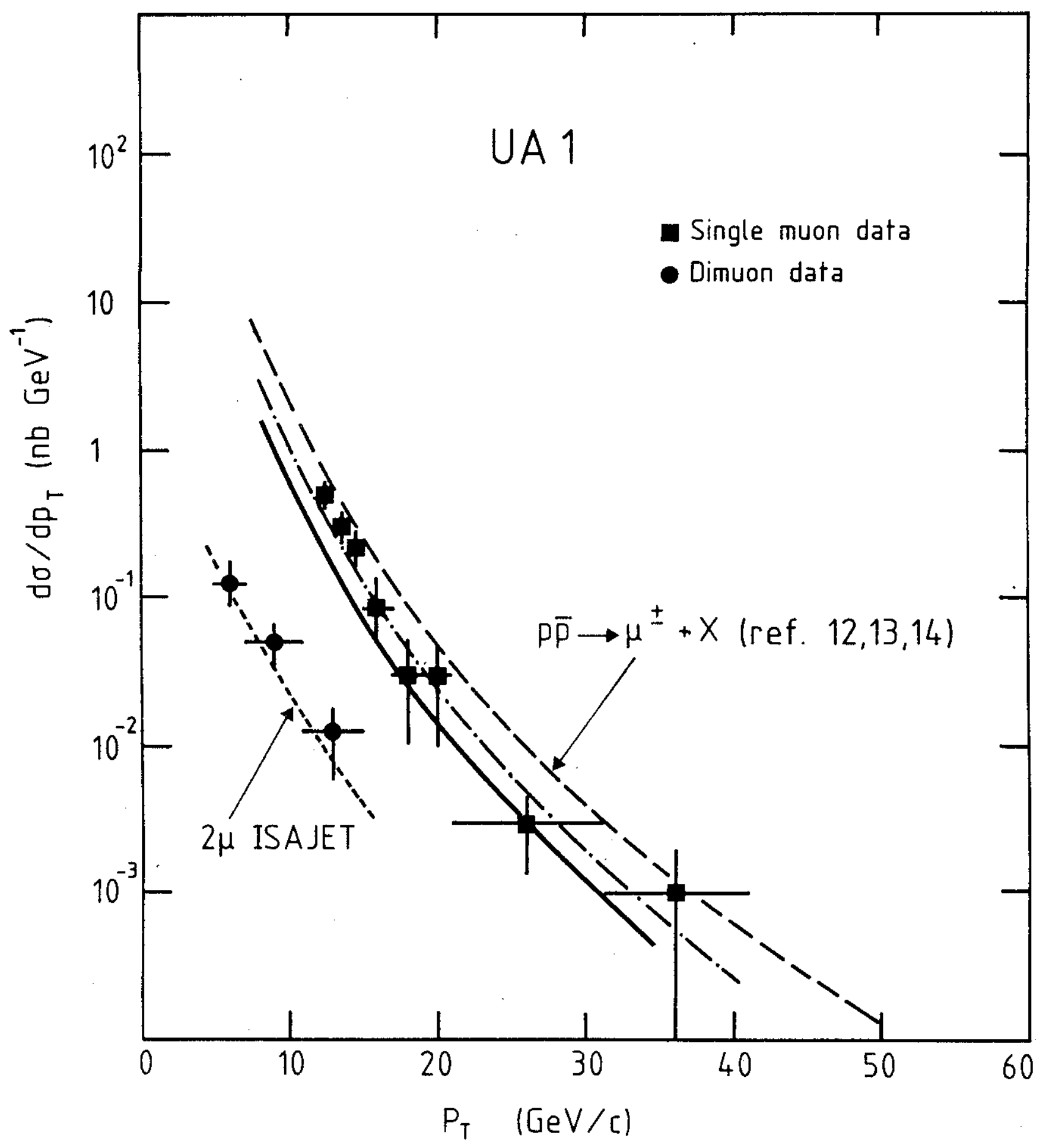

Fig. 5 


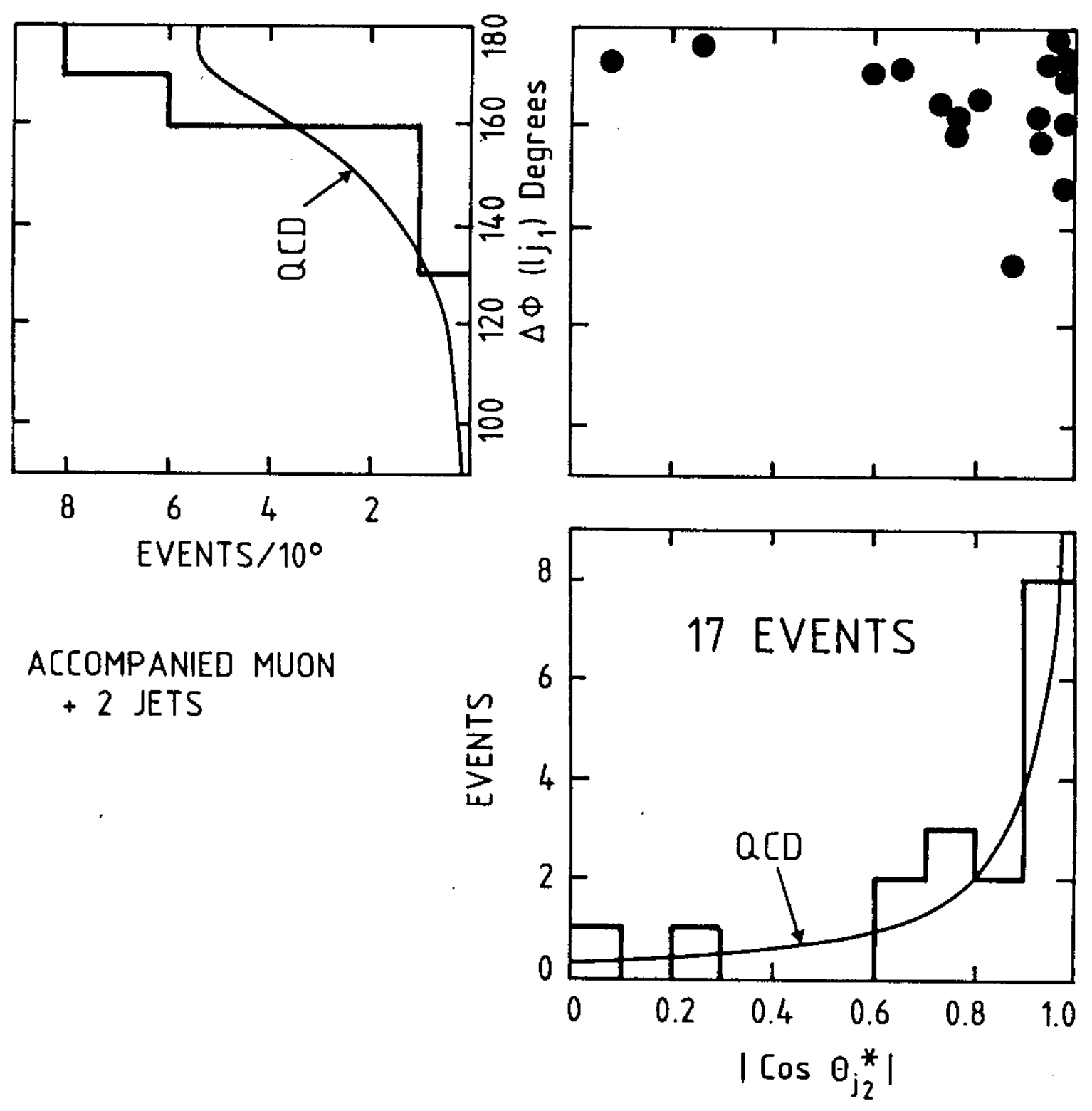

Fig. $6 a$ 


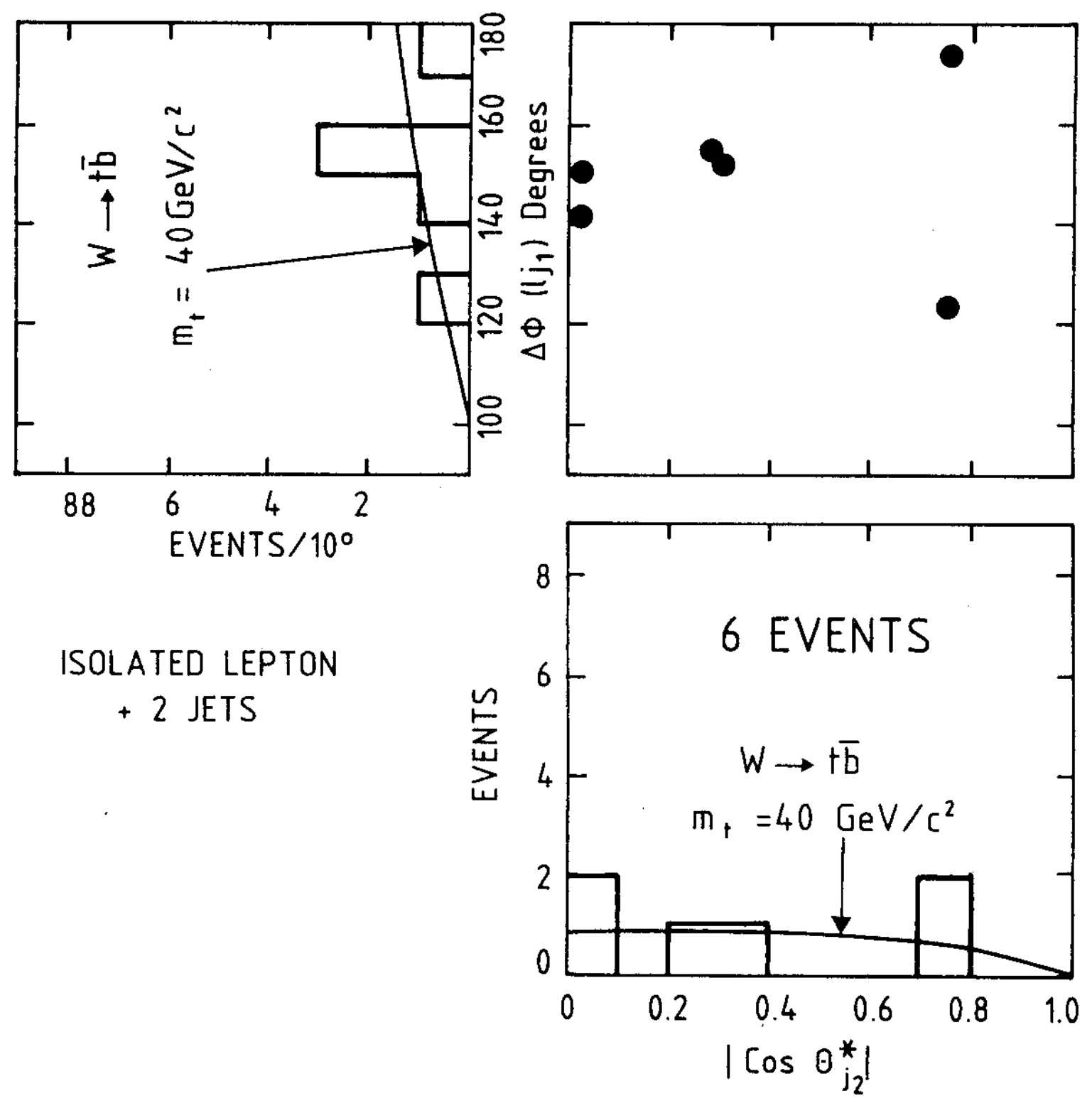

Fig. 6b 


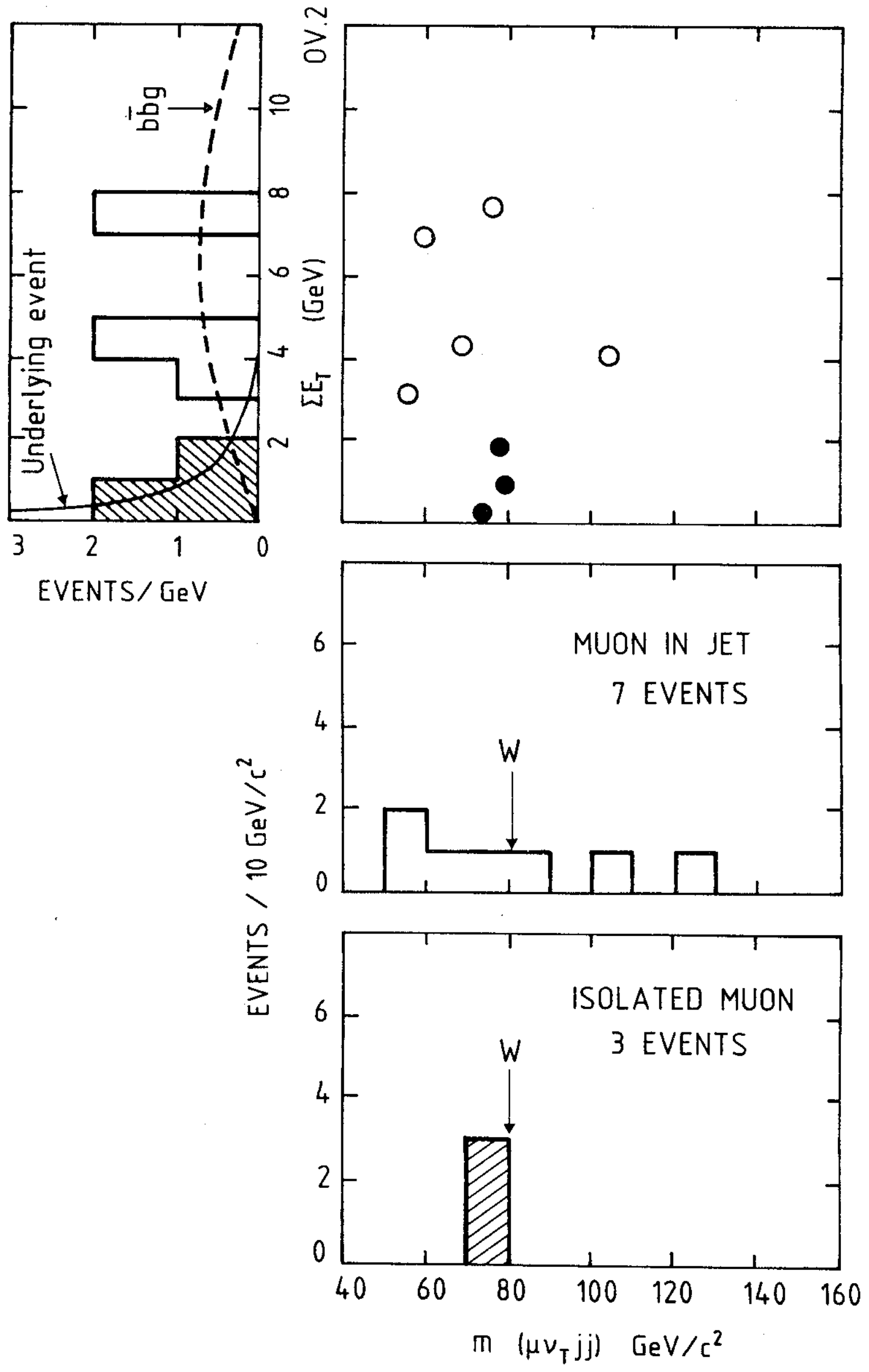

Fig. $7 a$ 


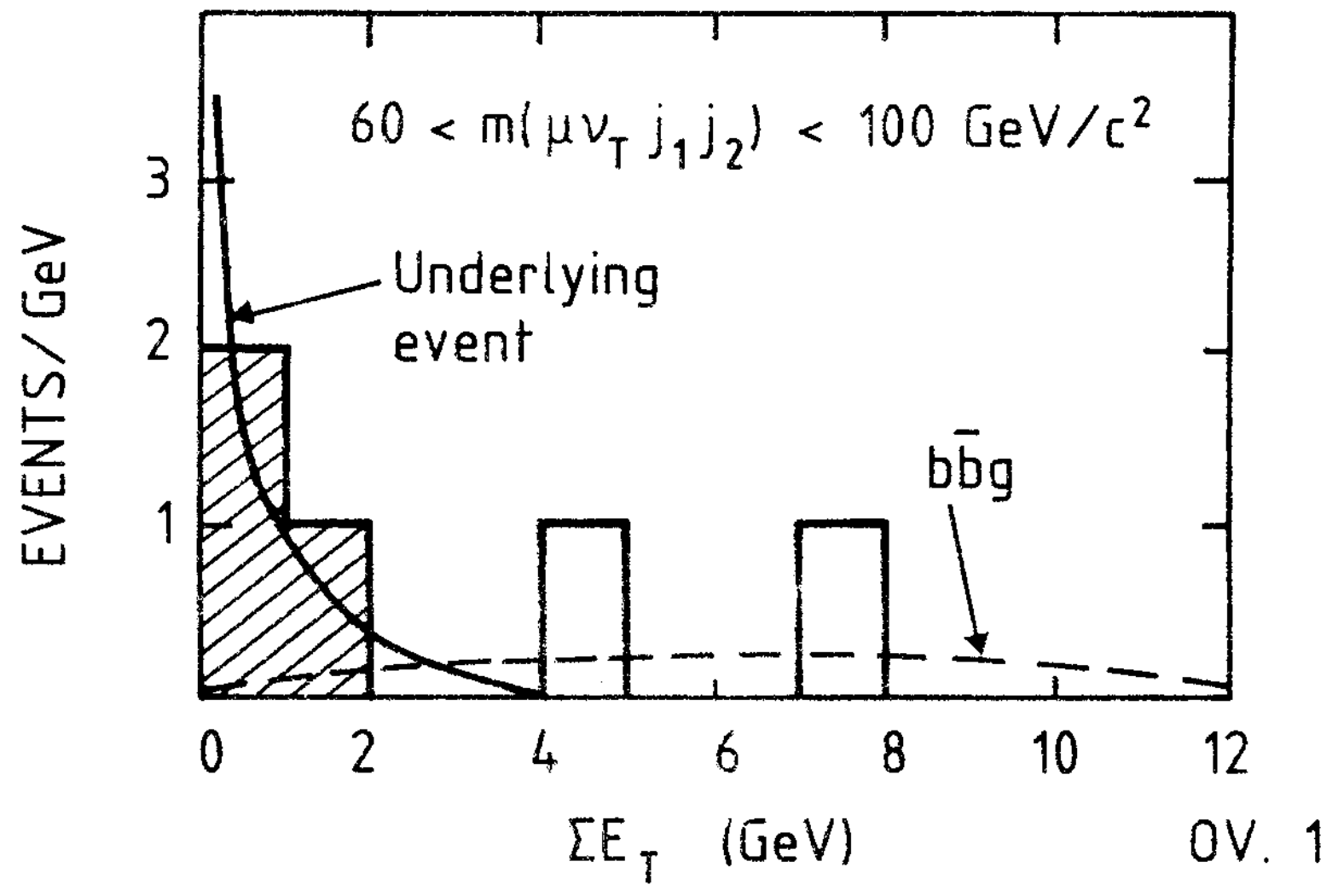

Fig. 75 


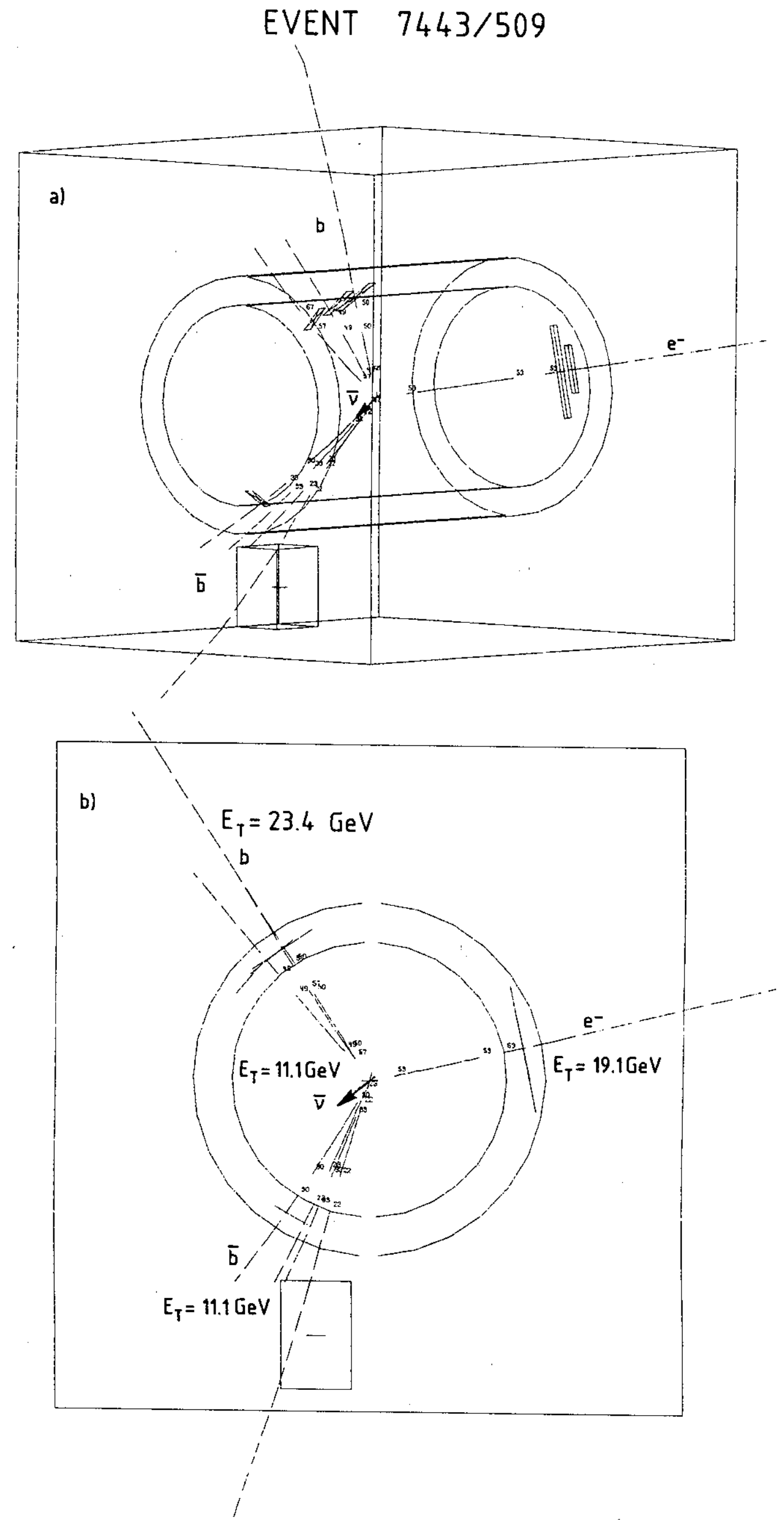

Fig. 8 


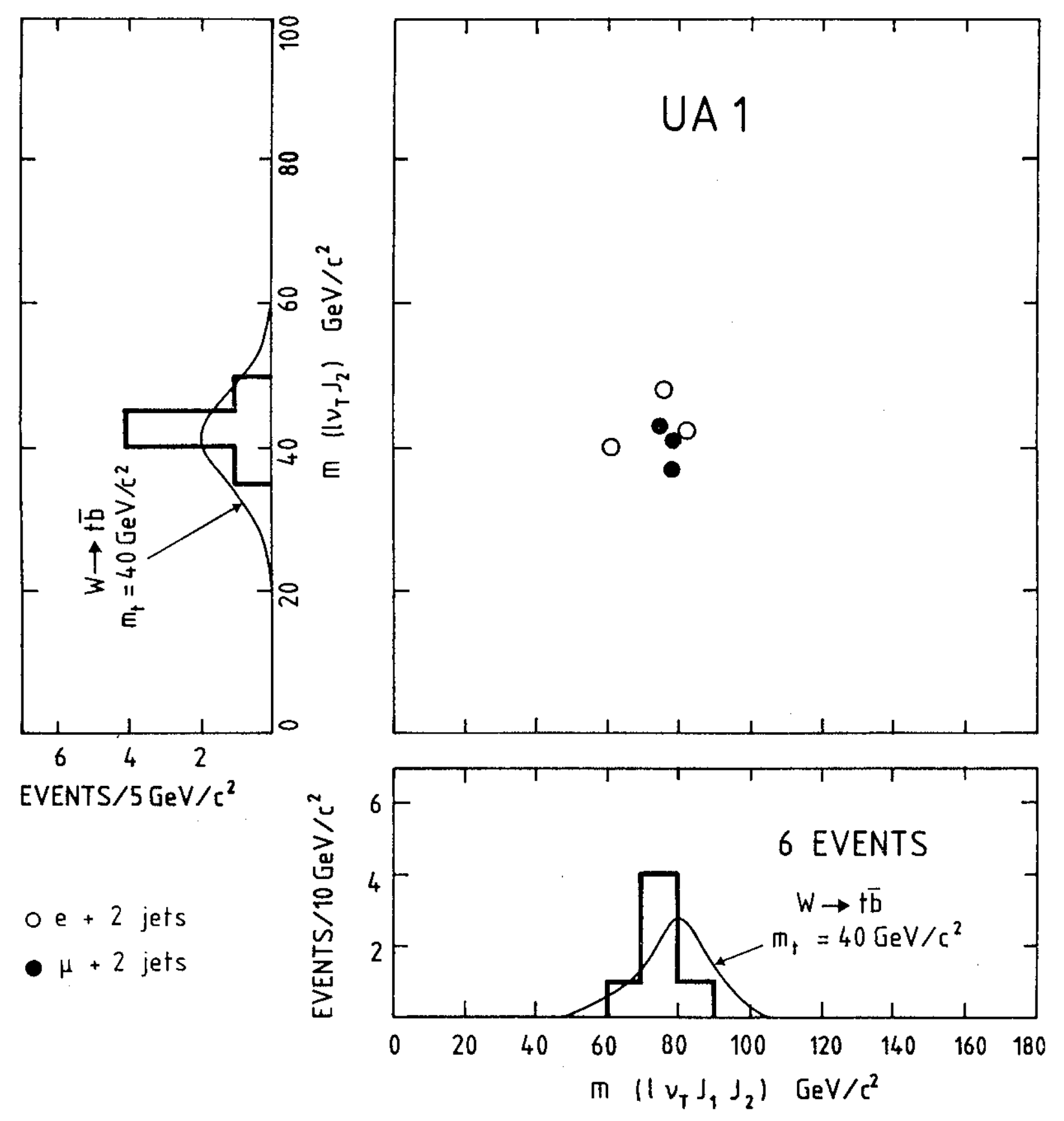

Fig. 9 


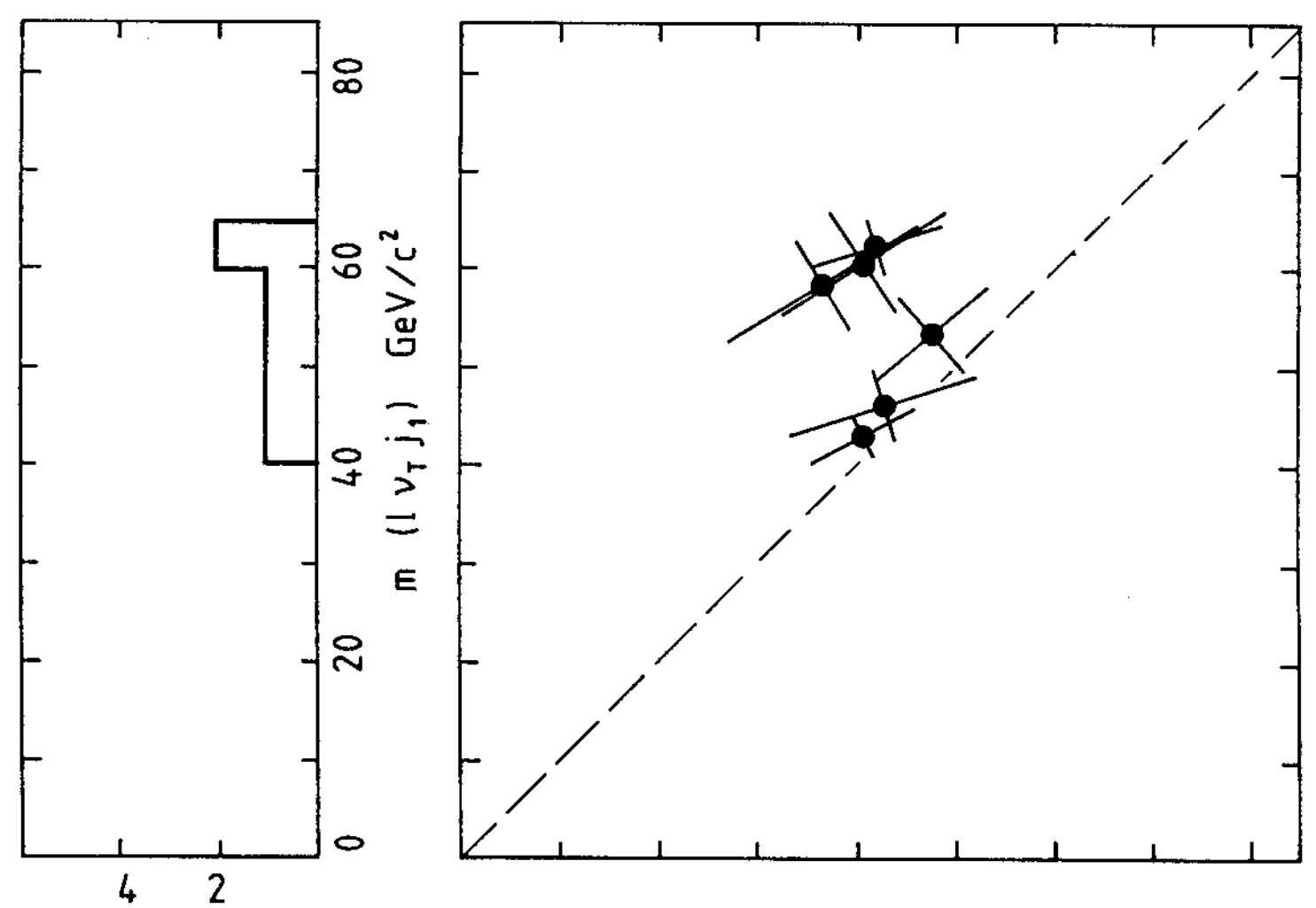

EVENTS $/ 5 \mathrm{GeV} / \mathrm{c}^{2}$

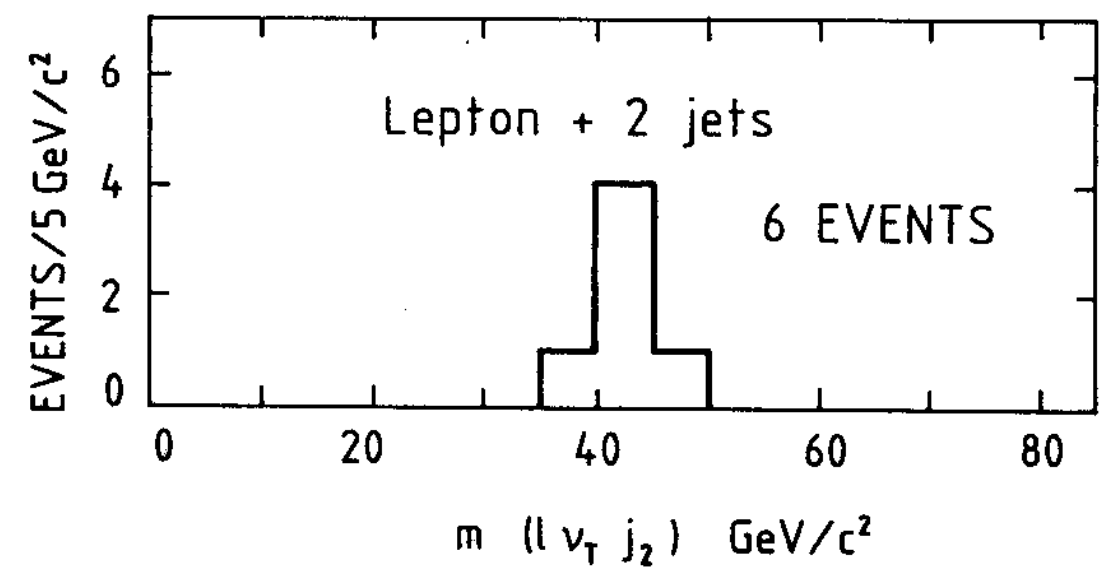

Fig. 10 

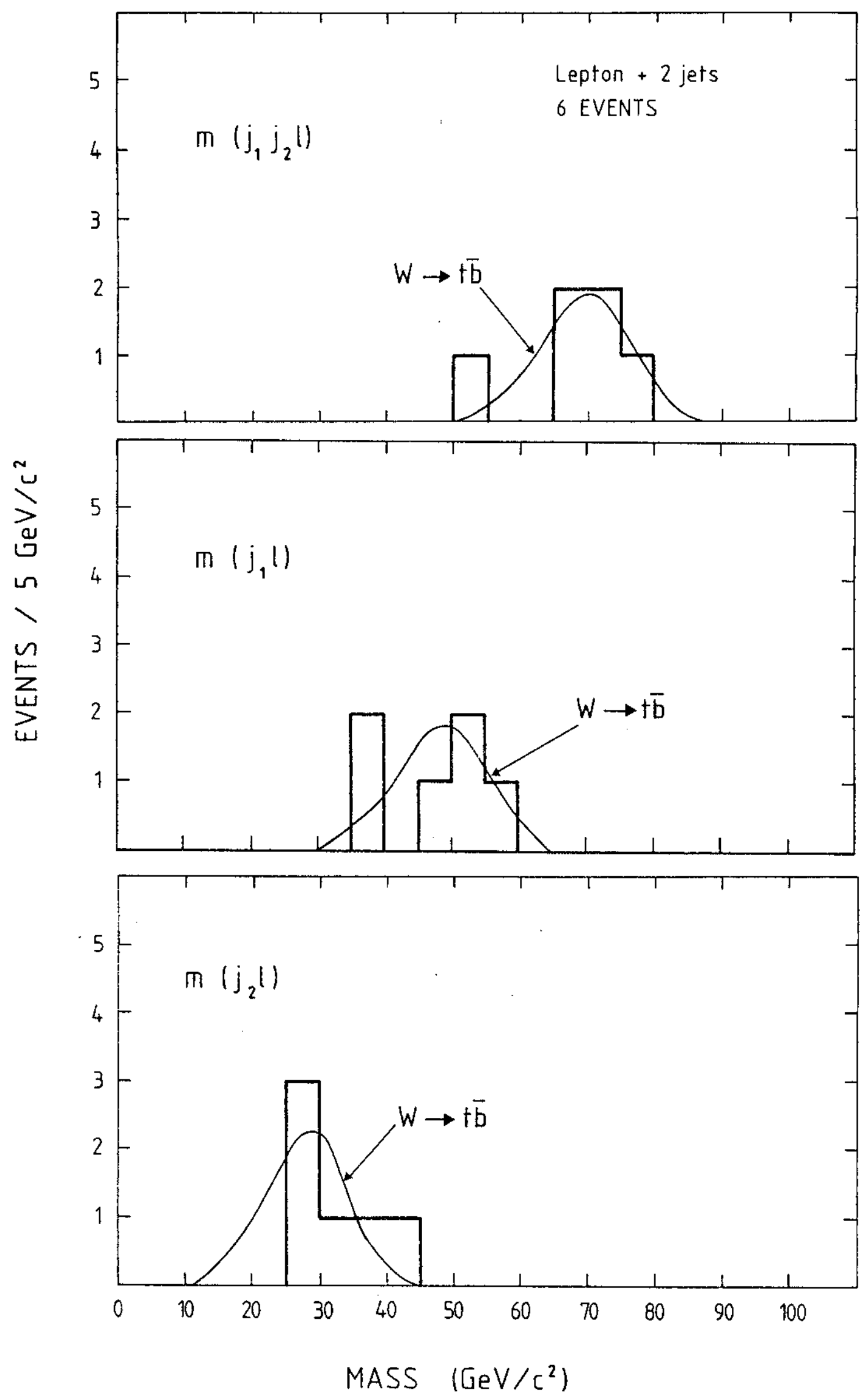

Fig. 11a 

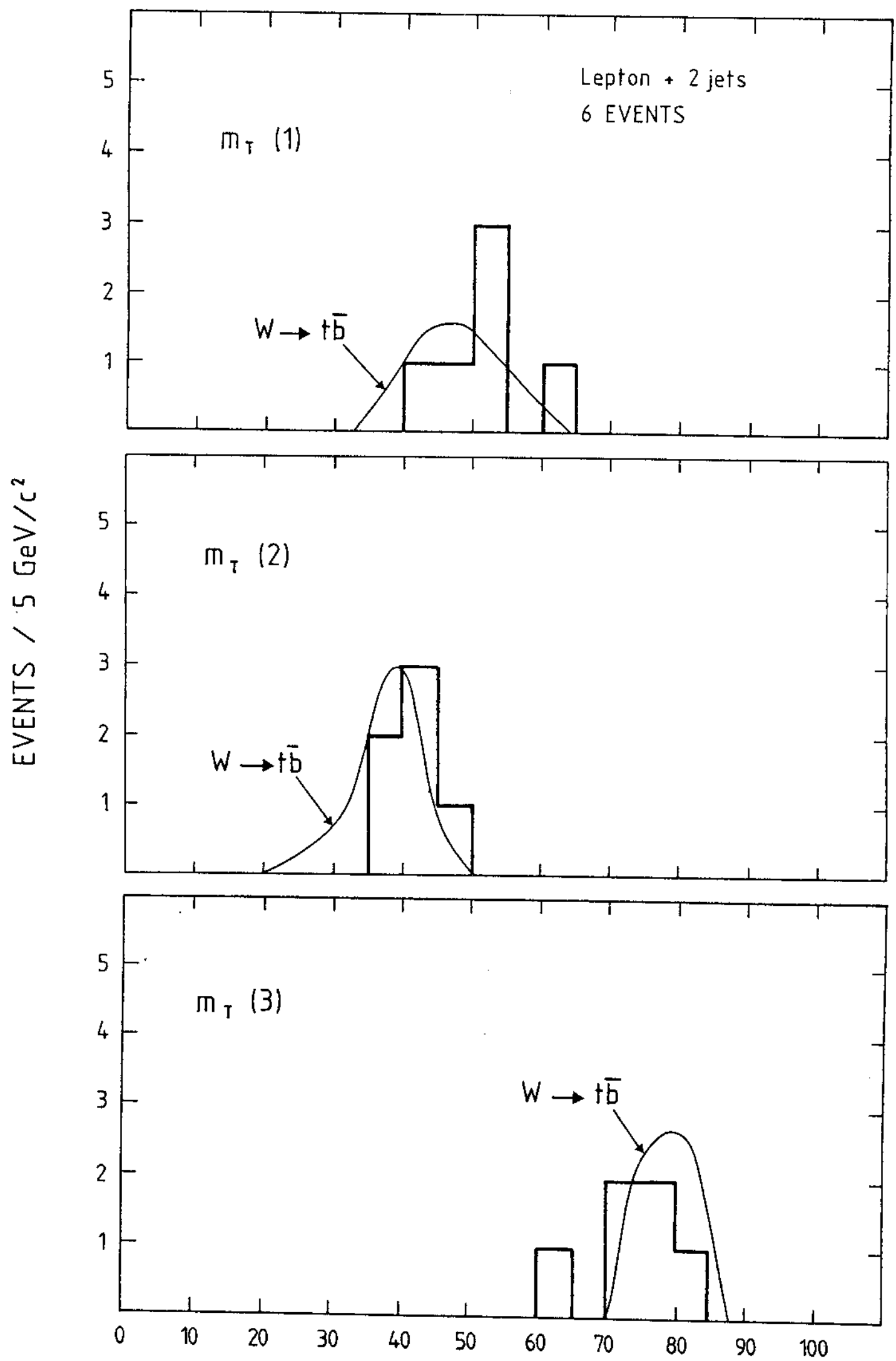

MASS $\left(\mathrm{GeV} / \mathrm{c}^{2}\right)$

Fig. 11b 